\title{
Ground state energy of dilute neutron matter at next-to-leading order in lattice chiral effective field theory
}

\author{
Evgeny Epelbaum ${ }^{a, b}$, Hermann Krebs ${ }^{b, a}$, Dean Lee ${ }^{c, b}$, Ulf-G. Meißner $^{b, a}$ \\ ${ }^{a}$ Institut für Kernphysik (IKP-3) and Jülich Center for Hadron Physics, \\ Forschungszentrum Jülich, D-52425 Jülich, Germany \\ ${ }^{b}$ Helmholtz-Institut für Strahlen- und Kernphysik (Theorie) \\ and Bethe Center for Theoretical Physics, \\ Universität Bonn, D-53115 Bonn, Germany \\ ${ }^{c}$ Department of Physics, North Carolina State University, Raleigh, NC 27695, USA
}

\begin{abstract}
We present lattice calculations for the ground state energy of dilute neutron matter at next-to-leading order in chiral effective field theory. This study follows a series of recent papers on low-energy nuclear physics using chiral effective field theory on the lattice. In this work we introduce an improved spin- and isospin-projected leading-order action which allows for a perturbative treatment of corrections at next-to-leading order and smaller estimated errors. Using auxiliary fields and Euclidean-time projection Monte Carlo, we compute the ground state of 8,12 , and 16 neutrons in a periodic cube, covering a density range from $2 \%$ to $10 \%$ of normal nuclear density.
\end{abstract}




\section{INTRODUCTION}

Chiral effective field theory for low-energy nucleons on the lattice has been investigated in several recent papers. In Ref. [1] chiral effective field theory was considered at leading order (LO) using two different lattice actions. These actions, $\mathrm{LO}_{1}$ and $\mathrm{LO}_{2}$, each include the leading-order interactions in Weinberg's power counting scheme [2, 3]. The difference is that in $\mathrm{LO}_{1}$ the nucleon-nucleon "contact" interactions are point-like while in $\mathrm{LO}_{2}$ they are smeared using a Gaussian function. These smeared interactions in $\mathrm{LO}_{2}$ were used to better reproduce $S$-wave phase shifts for nucleon momenta up to the pion mass. If the effective field theory expansion is converging properly then low-energy physical observables computed using $\mathrm{LO}_{1}$ and $\mathrm{LO}_{2}$ should agree up to differences of the size of next-to-leading order (NLO) corrections. Similarly when NLO corrections are included, agreement should be comparable to corrections at next-to-next-to-leading order (NNLO).

In Ref. [4] the spherical wall method [5] was used to calculate nucleon-nucleon scattering phase shifts and the $S$ - $D$ mixing angle for $\mathrm{LO}_{1}$ and $\mathrm{LO}_{2}$ at spatial lattice spacing $a=(100$

$\mathrm{MeV})^{-1}$ and temporal lattice spacing $a_{t}=(70 \mathrm{MeV})^{-1}$. In a companion paper [6] the same $\mathrm{LO}_{2}$ lattice action was reproduced using auxiliary fields, and the ground state energy of dilute neutron matter was calculated using projection Monte Carlo at densities ranging from $2 \%$ to $8 \%$ of normal nuclear density. For each Monte Carlo configuration next-toleading order corrections were computed using first-order perturbation theory. Simulations using the lattice action $\mathrm{LO}_{1}$ were also attempted, however strong complex phase oscillations prevented an accurate calculation.

Ground state energy results using the $\mathrm{LO}_{2}$ action at leading order and next-to-leading order are shown in Fig. 1. The energy is plotted as a fraction of the ground state energy for non-interacting neutrons at the same Fermi momentum $k_{F}$. For $k_{F}$ less than $100 \mathrm{MeV}$, the difference between results at leading order and next-to-leading order is small enough that the convergence of the effective theory appears reliable. However for $k_{F}$ greater than $100 \mathrm{MeV}$ the difference is relatively large, and the perturbative treatment of NLO corrections seems questionable. The analysis in Ref. [6] found that much of the difference between the $\mathrm{LO}_{2}$ and $\mathrm{NLO}_{2}$ results could be ascribed to differences in the $P$-wave phase shifts. Although helpful in $S$-wave channels, the Gaussian smearing used in $\mathrm{LO}_{2}$ produces unphysical attractive forces in each $P$-wave channel which must be cancelled at next-to-leading order. In this 


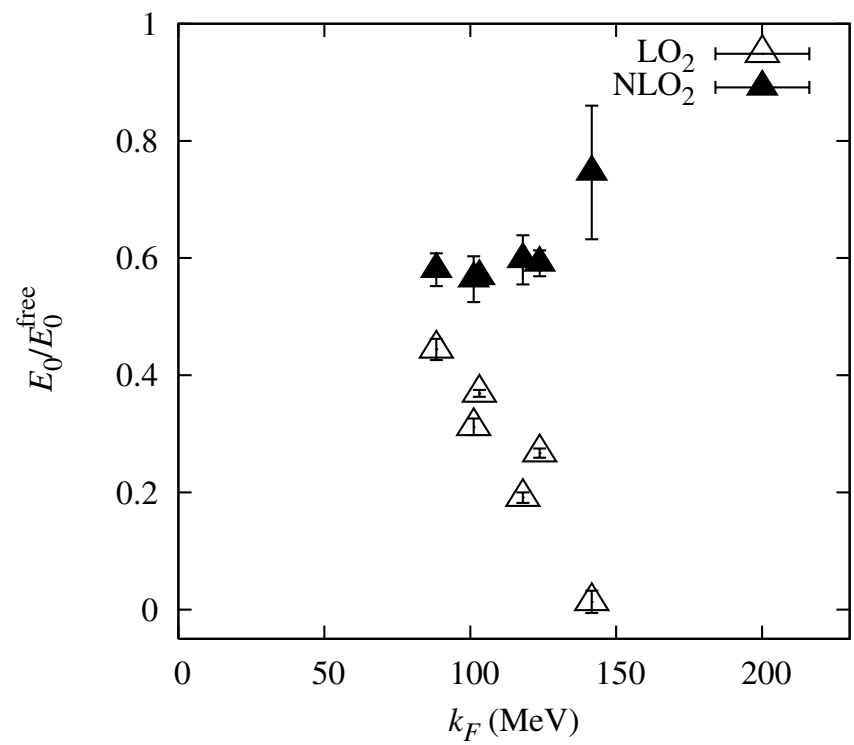

FIG. 1: Ground state energy ratio $E_{0} / E_{0}^{\text {free }}$ for dilute neutron matter versus Fermi momentum $k_{F}$ for $\mathrm{LO}_{2}$ and $\mathrm{NLO}_{2}[6]$.

paper we introduce a new leading-order action $\mathrm{LO}_{3}$ that solves this problem. The new action equals $\mathrm{LO}_{2}$ in each $S$-wave channel but matches $\mathrm{LO}_{1}$ in each $P$-wave channel. We construct the new action using projection operators for the spin-singlet/isospin-triplet and spin-triplet/isospin-singlet channels.

The paper is organized as follows. We first review the effective potential for chiral effective field theory up to next-to-leading order and simplifications that can be made at low cutoff momentum. We also summarize the lattice transfer matrix formalism for $\mathrm{LO}_{1}$ and $\mathrm{LO}_{2}$. The new action $\mathrm{LO}_{3}$ is then introduced and phase shifts and the $S$ - $D$ mixing angle are computed up to next-to-leading order. After this we rewrite the $\mathrm{LO}_{3}$ transfer matrix in terms of one-body interactions with auxiliary fields. This allows us to simulate the ground state of the many-neutron system up to next-to-leading order using projection Monte Carlo. We compare the new results obtained using $\mathrm{LO}_{3}$ and $\mathrm{NLO}_{3}$ with the $\mathrm{LO}_{2}$ and $\mathrm{NLO}_{2}$ results from Ref. [6] and other published data in the literature. We also analyze the ground state energy ratio $E_{0} / E_{0}^{\text {free }}$ as an expansion near the unitarity limit. 


\section{CHIRAL EFFECTIVE FIELD THEORY}

\section{A. Effective potential}

In our notation $\vec{q}$ denotes the $t$-channel momentum transfer for nucleon-nucleon scattering and $\vec{k}$ is the $u$-channel exchanged momentum transfer. At leading order in the Weinberg power-counting scheme [2, 3] the nucleon-nucleon effective potential includes two independent contact terms and instantaneous one-pion exchange (OPEP),

$$
\begin{gathered}
V_{\mathrm{LO}}=V^{(0)}+V^{\mathrm{OPEP}}, \\
V^{(0)}=C_{S}+C_{T}\left(\vec{\sigma}_{1} \cdot \vec{\sigma}_{2}\right), \\
V^{\mathrm{OPEP}}=-\left(\frac{g_{A}}{2 f_{\pi}}\right)^{2} \boldsymbol{\tau}_{1} \cdot \boldsymbol{\tau}_{2} \frac{\left(\vec{\sigma}_{1} \cdot \vec{q}\right)\left(\vec{\sigma}_{2} \cdot \vec{q}\right)}{q^{2}+m_{\pi}^{2}} .
\end{gathered}
$$

The vector arrow in $\vec{\sigma}$ signifies the three-vector index for spin, and the boldface for $\boldsymbol{\tau}$ signifies the three-vector index for isospin. For physical constants we take $m=938.92 \mathrm{MeV}$ as the nucleon mass, $m_{\pi}=138.08 \mathrm{MeV}$ as the pion mass, $f_{\pi}=93 \mathrm{MeV}$ as the pion decay constant, and $g_{A}=1.26$ as the nucleon axial charge.

At next-to-leading order the effective potential has seven independent contact terms carrying two powers of momentum, corrections to the two LO contact terms, and instantaneous two-pion exchange (TPEP) [7, 8, 9, 10, 11]. Following the notation of Ref. [10, 11] we have

$$
V_{\mathrm{NLO}}=V_{\mathrm{LO}}+\Delta V^{(0)}+V^{(2)}+V_{\mathrm{NLO}}^{\mathrm{TPEP}} .
$$

The NLO contact interactions are given by

$$
\begin{gathered}
\Delta V^{(0)}=\Delta C_{S}+\Delta C_{T}\left(\vec{\sigma}_{1} \cdot \vec{\sigma}_{2}\right), \\
V^{(2)}=C_{1} q^{2}+C_{2} k^{2}+\left(C_{3} q^{2}+C_{4} k^{2}\right)\left(\vec{\sigma}_{1} \cdot \vec{\sigma}_{2}\right)+i C_{5} \frac{1}{2}\left(\vec{\sigma}_{1}+\vec{\sigma}_{2}\right) \cdot(\vec{q} \times \vec{k}) \\
+C_{6}\left(\vec{\sigma}_{1} \cdot \vec{q}\right)\left(\vec{\sigma}_{2} \cdot \vec{q}\right)+C_{7}\left(\vec{\sigma}_{1} \cdot \vec{k}\right)\left(\vec{\sigma}_{2} \cdot \vec{k}\right),
\end{gathered}
$$

and the NLO two-pion exchange potential is [12, 13]

$$
\begin{aligned}
V_{\mathrm{NLO}}^{\mathrm{TPEP}} & =-\frac{\boldsymbol{\tau}_{1} \cdot \boldsymbol{\tau}_{2}}{384 \pi^{2} f_{\pi}^{4}} L(q)\left[4 m_{\pi}^{2}\left(5 g_{A}^{4}-4 g_{A}^{2}-1\right)+q^{2}\left(23 g_{A}^{4}-10 g_{A}^{2}-1\right)+\frac{48 g_{A}^{4} m_{\pi}^{4}}{4 m_{\pi}^{2}+q^{2}}\right] \\
& -\frac{3 g_{A}^{4}}{64 \pi^{2} f_{\pi}^{4}} L(q)\left[\left(\vec{q} \cdot \vec{\sigma}_{1}\right)\left(\vec{q} \cdot \vec{\sigma}_{2}\right)-q^{2}\left(\vec{\sigma}_{1} \cdot \vec{\sigma}_{2}\right)\right],
\end{aligned}
$$


where

$$
L(q)=\frac{1}{2 q} \sqrt{4 m_{\pi}^{2}+q^{2}} \ln \frac{\sqrt{4 m_{\pi}^{2}+q^{2}}+q}{\sqrt{4 m_{\pi}^{2}+q^{2}}-q} .
$$

Recent reviews of chiral effective field theory can be found in Ref. [14, 15, 16, 17].

\section{B. Power counting and cutoff momentum}

There have been a number of studies on the short-distance behavior of the one-pion exchange potential and the consistency of the Weinberg power-counting scheme. An alternative scheme known as KSW power counting was proposed [18, 19, 20]. This scheme is based on a perturbative treatment of the one-pion exchange potential and allows for systematic control of ultraviolet divergences in the effective theory. Unfortunately convergence at higher orders was found to be poor in some partial waves for momenta comparable to the pion mass [21].

Other power counting alternatives have also been proposed. In one scheme the leading $f_{\pi}^{-2} r^{-3}$ short-distance singularity is treated non-perturbatively while the remainder of the one-pion potential is introduced as a perturbative expansion in powers of $m_{\pi}$ [22]. More recently a different power counting modification was proposed in which one-pion exchange is treated non-perturbatively in lower angular momentum channels along with higher-derivative counterterms promoted to leading order [23]. Further investigations of this approach in higher partial waves and power counting with one-pion exchange were considered in Ref. [24, 25].

On the lattice the ultraviolet momentum cutoff is inversely proportional to the lattice spacing, $\Lambda=\pi / a$. For simple calculations of two-nucleon scattering on the lattice we could take any lattice spacing satisfying $\Lambda \gg m_{\pi}$. However in few- and many-nucleon calculations where we use Euclidean-time projection and auxiliary-field Monte Carlo methods, severe numerical problems appear when $\Lambda$ is very large. In some attractive channels the problem is due to spurious deeply-bound states which may appear at sufficiently large $\Lambda$. In other channels one faces the problem due to short-range hard-core repulsion at very large $\Lambda$. This is manifested as sign or complex phase oscillations which scale exponentially with system size and strength of the repulsive interaction.

To avoid these problems we consider lattice simulations where the cutoff momentum is only a few times the pion mass. In this study we take $\Lambda=314 \mathrm{MeV} \approx 2.3 m_{\pi}$, corresponding 
with $a^{-1}=100 \mathrm{MeV}$. For this low cutoff scale the advantages of the alternative powercounting schemes discussed above are numerically insignificant [26], and so we use standard Weinberg power counting. For nearly all $|q|<\Lambda$ we can expand the two-pion exchange potential in powers of $q^{2} /\left(4 m_{\pi}^{2}\right)$,

$$
\begin{gathered}
L(q)=1+\frac{1}{3} \frac{q^{2}}{4 m_{\pi}^{2}}+\cdots \\
\frac{4 m_{\pi}^{2}}{4 m_{\pi}^{2}+q^{2}} L(q)=1-\frac{2}{3} \frac{q^{2}}{4 m_{\pi}^{2}}+\cdots \\
V_{\mathrm{NLO}}^{\mathrm{TPEP}}=-\frac{\boldsymbol{\tau}_{1} \cdot \boldsymbol{\tau}_{2}}{384 \pi^{2} f_{\pi}^{4}}\left[4 m_{\pi}^{2}\left(8 g_{A}^{4}-4 g_{A}^{2}-1\right)+\frac{2}{3} q^{2}\left(34 g_{A}^{4}-17 g_{A}^{2}-2\right)+O\left(\left(\frac{q^{2}}{4 m_{\pi}^{2}}\right)^{2}\right)\right] \\
-\frac{3 g_{A}^{4}}{64 \pi^{2} f_{\pi}^{4}}\left[\left(\vec{q} \cdot \vec{\sigma}_{1}\right)\left(\vec{q} \cdot \vec{\sigma}_{2}\right)-q^{2}\left(\vec{\sigma}_{1} \cdot \vec{\sigma}_{2}\right)\right]\left[1+O\left(\frac{q^{2}}{4 m_{\pi}^{2}}\right)\right] .
\end{gathered}
$$

This expansion fails to converge only for values of $q$ near the cutoff scale $\Lambda \approx 2.3 m_{\pi}$, where the effective theory is already problematic due to large cutoff effects of size $O\left(q^{2} / \Lambda^{2}\right)$. There is no reason to keep the full non-local structure of $V_{\mathrm{NLO}}^{\mathrm{TPEP}}$ at this lattice spacing. Instead we simply use

$$
\begin{gathered}
V_{\mathrm{LO}}=V^{(0)}+V^{\mathrm{OPEP}} \\
V_{\mathrm{NLO}}=V_{\mathrm{LO}}+\Delta V^{(0)}+V^{(2)}
\end{gathered}
$$

where the terms in Eq. (11) with up to two powers of $q$ are absorbed as a redefinition of the coefficients $\Delta V^{(0)}$ and $V^{(2)}$. This same approach can be applied to the two-pion exchange potential at next-to-next-to-leading order and higher-order $n$-pion exchange potentials.

\section{LATTICE FORMALISM}

\section{A. Lattice notation}

In this paper we assume exact isospin symmetry and neglect electromagnetic interactions. We use $\vec{n}$ to represent integer-valued lattice vectors on a three-dimensional spatial lattice and either $\vec{p}, \vec{q}$, or $\vec{k}$ to represent integer-valued momentum lattice vectors. $\hat{l}=\hat{1}, \hat{2}, \hat{3}$ are unit lattice vectors in the spatial directions, $a$ is the spatial lattice spacing, and $L$ is the length of the cubic spatial lattice in each direction. We use the Euclidean transfer matrix formalism defined in [1] with lattice time step $a_{t}$, and the integer $n_{t}$ labels the time 
steps. We define $\alpha_{t}$ as the ratio between lattice spacings, $\alpha_{t}=a_{t} / a$. Throughout we use dimensionless parameters and operators, which correspond with physical values multiplied by the appropriate power of $a$. Final results are presented in physical units with the corresponding unit stated explicitly. As in [1] the spatial lattice spacing is $a=(100$ $\mathrm{MeV})^{-1}$ and temporal lattice spacing is $a_{t}=(70 \mathrm{MeV})^{-1}$.

We use $a$ and $a^{\dagger}$ to denote annihilation and creation operators. To avoid confusion we make explicit in our lattice notation all spin and isospin indices using

$$
\begin{aligned}
& a_{0,0}=a_{\uparrow, p}, \quad a_{0,1}=a_{\uparrow, n}, \\
& a_{1,0}=a_{\downarrow, p}, \quad a_{1,1}=a_{\downarrow, n} .
\end{aligned}
$$

The first subscript is for spin and the second subscript is for isospin. We use $\tau_{I}$ with $I=1,2,3$ to represent Pauli matrices acting in isospin space and $\sigma_{S}$ with $S=1,2,3$ to represent Pauli matrices acting in spin space. We also use the letters $S$ and $I$ to denote the total spin and total isospin for the two-nucleon system. The intended meaning in each case should be clear from the context. We use the eight vertices of a unit cube on the lattice to define spatial derivatives. For each spatial direction $l=1,2,3$ and any lattice function $f(\vec{n})$, let

$$
\Delta_{l} f(\vec{n})=\frac{1}{4} \sum_{\nu_{1}, \nu_{2}, \nu_{3}=0,1}(-1)^{\nu_{l}+1} f(\vec{n}+\vec{\nu}), \quad \vec{\nu}=\nu_{1} \hat{1}+\nu_{2} \hat{2}+\nu_{3} \hat{3} .
$$

We also define the double spatial derivative along direction $l$,

$$
\nabla_{l}^{2} f(\vec{n})=f(\vec{n}+\hat{l})+f(\vec{n}-\hat{l})-2 f(\vec{n})
$$

\section{B. Densities and current densities}

We define the local density of nucleons at lattice site $\vec{n}$,

$$
\rho^{a^{\dagger}, a}(\vec{n})=\sum_{i, j=0,1} a_{i, j}^{\dagger}(\vec{n}) a_{i, j}(\vec{n})
$$

This is invariant under Wigner's SU(4) symmetry transforming all spin and isospin degrees of freedom [27]. Similarly we define a local spin density for $S=1,2,3$,

$$
\rho_{S}^{a^{\dagger}, a}(\vec{n})=\sum_{i, j, i^{\prime}=0,1} a_{i, j}^{\dagger}(\vec{n})\left[\sigma_{S}\right]_{i i^{\prime}} a_{i^{\prime}, j}(\vec{n})
$$


isospin density for $I=1,2,3$,

$$
\rho_{I}^{a^{\dagger}, a}(\vec{n})=\sum_{i, j, j^{\prime}=0,1} a_{i, j}^{\dagger}(\vec{n})\left[\tau_{I}\right]_{j j^{\prime}} a_{i, j^{\prime}}(\vec{n})
$$

and spin-isospin density for $S, I=1,2,3$,

$$
\rho_{S, I}^{a^{\dagger}, a}(\vec{n})=\sum_{i, j, i^{\prime}, j^{\prime}=0,1} a_{i, j}^{\dagger}(\vec{n})\left[\sigma_{S}\right]_{i i^{\prime}}\left[\tau_{I}\right]_{j j^{\prime}} a_{i^{\prime}, j^{\prime}}(\vec{n})
$$

For each static density we also have an associated current density. Similar to the definition of the lattice derivative $\Delta_{l}$ in Eq. (16), we use the eight vertices of a unit cube,

$$
\vec{\nu}=\nu_{1} \hat{1}+\nu_{2} \hat{2}+\nu_{3} \hat{3}
$$

for $\nu_{1}, \nu_{2}, \nu_{3}=0,1$. Let $\vec{\nu}(-l)$ for $l=1,2,3$ be the result of reflecting the $l^{\text {th }}$-component of $\vec{\nu}$ about the center of the cube,

$$
\vec{\nu}(-l)=\vec{\nu}+\left(1-2 \nu_{l}\right) \hat{l}
$$

Omitting factors of $i$ and $1 / m$, we can write the $l^{\text {th }}$-component of the SU(4)-invariant current density as

$$
\Pi_{l}^{a^{\dagger}, a}(\vec{n})=\frac{1}{4} \sum_{\nu_{1}, \nu_{2}, \nu_{3}=0,1} \sum_{i, j=0,1}(-1)^{\nu_{l}+1} a_{i, j}^{\dagger}(\vec{n}+\vec{\nu}(-l)) a_{i, j}(\vec{n}+\vec{\nu}) .
$$

Similarly the $l^{\text {th }}$-component of spin current density is

$$
\Pi_{l, S}^{a^{\dagger}, a}(\vec{n})=\frac{1}{4} \sum_{\nu_{1}, \nu_{2}, \nu_{3}=0,1} \sum_{i, j, i^{\prime}=0,1}(-1)^{\nu_{l}+1} a_{i, j}^{\dagger}(\vec{n}+\vec{\nu}(-l))\left[\sigma_{S}\right]_{i i^{\prime}} a_{i^{\prime}, j}(\vec{n}+\vec{\nu}),
$$

$l^{\text {th }}$-component of isospin current density is

$$
\Pi_{l, I}^{a^{\dagger}, a}(\vec{n})=\frac{1}{4} \sum_{\nu_{1}, \nu_{2}, \nu_{3}=0,1} \sum_{i, j, j^{\prime}=0,1}(-1)^{\nu_{l}+1} a_{i, j}^{\dagger}(\vec{n}+\vec{\nu}(-l))\left[\tau_{I}\right]_{j j^{\prime}} a_{i, j^{\prime}}(\vec{n}+\vec{\nu}),
$$

and $l^{\text {th }}$-component of spin-isospin current density is

$$
\Pi_{l, S, I}^{a^{\dagger}, a}(\vec{n})=\frac{1}{4} \sum_{\nu_{1}, \nu_{2}, \nu_{3}=0,1} \sum_{i, j, i^{\prime}, j^{\prime}=0,1}(-1)^{\nu_{l}+1} a_{i, j}^{\dagger}(\vec{n}+\vec{\nu}(-l))\left[\sigma_{S}\right]_{i i^{\prime}}\left[\tau_{I}\right]_{j j^{\prime}} a_{i^{\prime}, j^{\prime}}(\vec{n}+\vec{\nu}) \text {. }
$$




\section{LATTICE ACTIONS}

\section{A. Instantaneous free pion action}

The lattice action for free pions with purely instantaneous propagation is

$$
S_{\pi \pi}\left(\pi_{I}\right)=\alpha_{t}\left(\frac{m_{\pi}^{2}}{2}+3\right) \sum_{\vec{n}, n_{t}, I} \pi_{I}\left(\vec{n}, n_{t}\right) \pi_{I}\left(\vec{n}, n_{t}\right)-\alpha_{t} \sum_{\vec{n}, n_{t}, I, l} \pi_{I}\left(\vec{n}, n_{t}\right) \pi_{I}\left(\vec{n}+\hat{l}, n_{t}\right),
$$

where $\pi_{I}$ is the pion field labelled with isospin index $I$. We note that pion fields at different time steps $n_{t}$ and $n_{t}^{\prime}$ are decoupled due to the omission of time derivatives. This decoupling among different time steps generates instantaneous propagation in one-pion exchange diagrams and eliminates radiative pion loops. It is convenient to define a rescaled pion field, $\pi_{I}^{\prime}$

$$
\begin{gathered}
\pi_{I}^{\prime}\left(\vec{n}, n_{t}\right)=\sqrt{q_{\pi}} \pi_{I}\left(\vec{n}, n_{t}\right), \\
q_{\pi}=\alpha_{t}\left(m_{\pi}^{2}+6\right) .
\end{gathered}
$$

Then

$$
S_{\pi \pi}\left(\pi_{I}^{\prime}\right)=\frac{1}{2} \sum_{\vec{n}, n_{t}, I} \pi_{I}^{\prime}\left(\vec{n}, n_{t}\right) \pi_{I}^{\prime}\left(\vec{n}, n_{t}\right)-\frac{\alpha_{t}}{q_{\pi}} \sum_{\vec{n}, n_{t}, I, l} \pi_{I}^{\prime}\left(\vec{n}, n_{t}\right) \pi_{I}^{\prime}\left(\vec{n}+\hat{l}, n_{t}\right) .
$$

In momentum space the action is

$$
S_{\pi \pi}\left(\pi_{I}^{\prime}\right)=\frac{1}{L^{3}} \sum_{I, \vec{k}} \pi_{I}^{\prime}\left(-\vec{k}, n_{t}\right) \pi_{I}^{\prime}\left(\vec{k}, n_{t}\right)\left[\frac{1}{2}-\frac{\alpha_{t}}{q_{\pi}} \sum_{l} \cos \left(\frac{2 \pi k_{l}}{L}\right)\right] .
$$

The instantaneous pion correlation function at spatial separation $\vec{n}$ is

$$
\begin{aligned}
\left\langle\pi_{I}^{\prime}\left(\vec{n}, n_{t}\right) \pi_{I}^{\prime}\left(\overrightarrow{0}, n_{t}\right)\right\rangle & \left.=\frac{\int D \pi_{I}^{\prime} \pi_{I}^{\prime}\left(\vec{n}, n_{t}\right) \pi_{I}^{\prime}\left(\overrightarrow{0}, n_{t}\right) \exp \left[-S_{\pi \pi}\right]}{\int D \pi_{I}^{\prime} \exp \left[-S_{\pi \pi}\right]} \text { (no sum on } I\right) \\
& =\frac{1}{L^{3}} \sum_{\vec{k}} e^{-i \frac{2 \pi}{L} \vec{k} \cdot \vec{n}} D_{\pi}(\vec{k}),
\end{aligned}
$$

where

$$
D_{\pi}(\vec{k})=\frac{1}{1-\frac{2 \alpha_{t}}{q_{\pi}} \sum_{l} \cos \left(\frac{2 \pi k_{l}}{L}\right)}
$$

\section{B. Transfer matrices for $\mathrm{LO}_{1}$ and $\mathrm{LO}_{2}$}

Roughly speaking, the Euclidean-time transfer matrix is the exponential of the Hamiltonian, $\exp (-H \Delta t)$, where $\Delta t$ equals one temporal lattice spacing. The normal-ordered 
transfer matrix for non-interacting nucleons is

$$
M_{\text {free }}=: \exp \left(-H_{\text {free }} \alpha_{t}\right):
$$

where the :: symbols indicate normal ordering. We use the $O\left(a^{4}\right)$-improved free lattice Hamiltonian,

$$
\begin{aligned}
H_{\text {free }} & =\frac{49}{12 m} \sum_{\vec{n}} \sum_{i, j=0,1} a_{i, j}^{\dagger}(\vec{n}) a_{i, j}(\vec{n}) \\
& -\frac{3}{4 m} \sum_{\vec{n}} \sum_{i, j=0,1} \sum_{l=1,2,3}\left[a_{i, j}^{\dagger}(\vec{n}) a_{i, j}(\vec{n}+\hat{l})+a_{i, j}^{\dagger}(\vec{n}) a_{i, j}(\vec{n}-\hat{l})\right] \\
& +\frac{3}{40 m} \sum_{\vec{n}} \sum_{i, j=0,1} \sum_{l=1,2,3}\left[a_{i, j}^{\dagger}(\vec{n}) a_{i, j}(\vec{n}+2 \hat{l})+a_{i, j}^{\dagger}(\vec{n}) a_{i, j}(\vec{n}-2 \hat{l})\right] \\
& -\frac{1}{180 m} \sum_{\vec{n}} \sum_{i, j=0,1} \sum_{l=1,2,3}\left[a_{i, j}^{\dagger}(\vec{n}) a_{i, j}(\vec{n}+3 \hat{l})+a_{i, j}^{\dagger}(\vec{n}) a_{i, j}(\vec{n}-3 \hat{l})\right] .
\end{aligned}
$$

Let us define the two-derivative pion correlator,

$$
\begin{aligned}
G_{S_{1} S_{2}}(\vec{n}) & \left.=\left\langle\Delta_{S_{1}} \pi_{I}^{\prime}\left(\vec{n}, n_{t}\right) \Delta_{S_{2}} \pi_{I}^{\prime}\left(\overrightarrow{0}, n_{t}\right)\right\rangle \quad \text { (no sum on } I\right) \\
& =\frac{1}{16} \sum_{\nu_{1}, \nu_{2}, \nu_{3}=0,1} \sum_{\nu_{1}^{\prime}, \nu_{2}^{\prime}, \nu_{3}^{\prime}=0,1}(-1)^{\nu_{S_{1}}}(-1)^{\nu_{S_{2}}^{\prime}}\left\langle\pi_{I}^{\prime}\left(\vec{n}+\vec{\nu}-\vec{\nu}^{\prime}, n_{t}\right) \pi_{I}^{\prime}\left(\overrightarrow{0}, n_{t}\right)\right\rangle
\end{aligned}
$$

With interactions included, the lattice transfer matrix $\mathrm{LO}_{1}$ has the form

$$
\begin{aligned}
M_{\mathrm{LO}_{1}}=: & \exp \left\{-H_{\text {free }} \alpha_{t}-\frac{1}{2} C \alpha_{t} \sum_{\vec{n}}\left[\rho^{a^{\dagger}, a}(\vec{n})\right]^{2}-\frac{1}{2} C_{I^{2}} \alpha_{t} \sum_{I} \sum_{\vec{n}}\left[\rho_{I}^{a^{\dagger}, a}(\vec{n})\right]^{2}\right. \\
& \left.+\frac{g_{A}^{2} \alpha_{t}^{2}}{8 f_{\pi}^{2} q_{\pi}} \sum_{S_{1}, S_{2}, I} \sum_{\vec{n}_{1}, \vec{n}_{2}} G_{S_{1} S_{2}}\left(\vec{n}_{1}-\vec{n}_{2}\right) \rho_{S_{1}, I}^{a^{\dagger}, a}\left(\vec{n}_{1}\right) \rho_{S_{2}, I}^{a^{\dagger}, a}\left(\vec{n}_{2}\right)\right\}:
\end{aligned}
$$

where $C$ is the coefficient of the Wigner $\mathrm{SU}(4)$-invariant contact interaction and $C_{I^{2}}$ is the coefficient of the isospin-dependent contact interaction. For the $S$-wave there are two independent channels corresponding with the spin-singlet/isospin-triplet and the spintriplet/isospin-singlet. To reproduce the physical scattering lengths in each channel we set $C_{S=0, I=1}=-5.021 \times 10^{-5} \mathrm{MeV}^{-2}$ and $C_{S=1, I=0}=-5.714 \times 10^{-5} \mathrm{MeV}^{-2}$ and use the relations

$$
\begin{gathered}
C=\left(3 C_{S=0, I=1}+C_{S=1, I=0}\right) / 4, \\
C_{I^{2}}=\left(C_{S=0, I=1}-C_{S=1, I=0}\right) / 4 .
\end{gathered}
$$


The $\mathrm{LO}_{2}$ transfer matrix is [1]

$$
\begin{aligned}
M_{\mathrm{LO}_{2}}=: & \exp \left\{-H_{\text {free }} \alpha_{t}-\frac{\alpha_{t}}{2 L^{3}} \sum_{\vec{q}} f(\vec{q})\left[C \rho^{a^{\dagger}, a}(\vec{q}) \rho^{a^{\dagger}, a}(-\vec{q})+C_{I^{2}} \sum_{I} \rho_{I}^{a^{\dagger}, a}(\vec{q}) \rho_{I}^{a^{\dagger}, a}(-\vec{q})\right]\right. \\
& \left.+\frac{g_{A}^{2} \alpha_{t}^{2}}{8 f_{\pi}^{2} q_{\pi}} \sum_{S_{1}, S_{2}, I} \sum_{\vec{n}_{1}, \vec{n}_{2}} G_{S_{1} S_{2}}\left(\vec{n}_{1}-\vec{n}_{2}\right) \rho_{S_{1}, I}^{a^{\dagger}, a}\left(\vec{n}_{1}\right) \rho_{S_{2}, I}^{a^{\dagger}, a}\left(\vec{n}_{2}\right)\right\}:
\end{aligned}
$$

where the momentum-dependent coefficient function $f(\vec{q})$ is defined as

$$
f(\vec{q})=f_{0}^{-1} \exp \left[-b \sum_{l}\left(1-\cos q_{l}\right)\right],
$$

and the normalization factor $f_{0}$ is determined by the condition

$$
f_{0}=\frac{1}{L^{3}} \sum_{\vec{q}} \exp \left[-b \sum_{l}\left(1-\cos q_{l}\right)\right] \text {. }
$$

As in Ref. [1] we use the value $b=0.6$. This gives approximately the correct average effective range for the two $S$-wave channels when $C$ and $C_{I^{2}}$ are tuned to produce the physical $S$-wave scattering lengths. We set $C_{S=0, I=1}=-3.414 \times 10^{-5} \mathrm{MeV}^{-2}$ and $C_{S=1, I=0}=-4.780 \times 10^{-5}$ $\mathrm{MeV}^{-2}$ and use the same relations Eq. (39) and (40). The momentum-dependent function $f(\vec{q})$ produces the Gaussian-smeared "contact" interactions discussed in the introduction.

The replacement of pointlike interactions in $\mathrm{LO}_{1}$ with Gaussian-smeared interactions in $\mathrm{LO}_{2}$ is similar to the lattice improvement program of Symanzik used in lattice QCD actions [28, 29]. There is a conceptual difference however since we are dealing with an effective field theory rather than a renormalizable field theory. The higher-order operators we consider do not only cancel lattice artifacts but also include higher-order interactions of the effective theory. In our lattice calculations the improved leading-order action is treated non-perturbatively while higher-order interactions are included as a perturbative expansion. The choice of improved action sets a dividing line between perturbative and non-perturbative interactions. This dividing line should be immaterial so long as the perturbative expansion converges. At any given order, lattice calculations using different improved actions should agree up to corrections the size of terms at next order.

\section{Transfer matrix for $\mathrm{LO}_{3}$}

The Gaussian smearing used in $\mathrm{LO}_{2}$ is useful in $S$-wave channels but produces unphysical attractive forces in $P$-wave channels. To avoid this problem we introduce a new leading- 
order action $\mathrm{LO}_{3}$ that equals $\mathrm{LO}_{2}$ in each $S$-wave channel but matches $\mathrm{LO}_{1}$ in each $P$ wave channel. We multiply the Gaussian-smeared "contact" interactions with projection operators for the spin-singlet/isospin-triplet channel, $P_{S=0, I=1}$, and the spin-triplet/isospinsinglet channel, $P_{S=1, I=0}$. If we assign labels to the two nucleons, $A$ and $B$, these projection operators are

$$
\begin{aligned}
& P_{S=0, I=1}=\left(\frac{1}{4}-\frac{1}{4} \sum_{S} \sigma_{S}^{A} \sigma_{S}^{B}\right)\left(\frac{3}{4}+\frac{1}{4} \sum_{I} \tau_{I}^{A} \tau_{I}^{B}\right), \\
& P_{S=1, I=0}=\left(\frac{3}{4}+\frac{1}{4} \sum_{S} \sigma_{S}^{A} \sigma_{S}^{B}\right)\left(\frac{1}{4}-\frac{1}{4} \sum_{I} \tau_{I}^{A} \tau_{I}^{B}\right) .
\end{aligned}
$$

We can define corresponding momentum-dependent density correlations,

$$
\begin{aligned}
V_{S=0, I=1}(\vec{q}) & =\frac{3}{32}: \rho^{a^{\dagger}, a}(\vec{q}) \rho^{a^{\dagger}, a}(-\vec{q}):-\frac{3}{32}: \sum_{S} \rho_{S}^{a^{\dagger}, a}(\vec{q}) \rho_{S}^{a^{\dagger}, a}(-\vec{q}): \\
& +\frac{1}{32}: \sum_{I} \rho_{I}^{a^{\dagger}, a}(\vec{q}) \rho_{I}^{a^{\dagger}, a}(-\vec{q}):-\frac{1}{32}: \sum_{S, I} \rho_{S, I}^{a^{\dagger}, a}(\vec{q}) \rho_{S, I}^{a^{\dagger}, a}(-\vec{q}): \\
V_{S=1, I=0}(\vec{q}) & =\frac{3}{32}: \rho^{a^{\dagger}, a}(\vec{q}) \rho^{a^{\dagger}, a}(-\vec{q}):+\frac{1}{32}: \sum_{S} \rho_{S}^{a^{\dagger}, a}(\vec{q}) \rho_{S}^{a^{\dagger}, a}(-\vec{q}): \\
& -\frac{3}{32}: \sum_{I} \rho_{I}^{a^{\dagger}, a}(\vec{q}) \rho_{I}^{a^{\dagger}, a}(-\vec{q}):-\frac{1}{32}: \sum_{S, I} \rho_{S, I}^{a^{\dagger}, a}(\vec{q}) \rho_{S, I}^{a^{\dagger}, a}(-\vec{q}):
\end{aligned}
$$

We use $V_{S=0, I=1}$ and $V_{S=1, I=0}$ to write the leading-order transfer matrix for $\mathrm{LO}_{3}$,

$$
\begin{aligned}
M_{\mathrm{LO}_{3}}=: & \exp \left\{-H_{\text {free }} \alpha_{t}-\frac{\alpha_{t}}{L^{3}} \sum_{\vec{q}} f(\vec{q})\left[C_{S=0, I=1} V_{S=0, I=1}(\vec{q})+C_{S=1, I=0} V_{S=1, I=0}(\vec{q})\right]\right. \\
& \left.+\frac{g_{A}^{2} \alpha_{t}^{2}}{8 f_{\pi}^{2} q_{\pi}} \sum_{S_{1}, S_{2}, I} \sum_{\vec{n}_{1}, \vec{n}_{2}} G_{S_{1} S_{2}}\left(\vec{n}_{1}-\vec{n}_{2}\right) \rho_{S_{1}, I}^{a^{\dagger}, a}\left(\vec{n}_{1}\right) \rho_{S_{2}, I}^{a^{\dagger}, a}\left(\vec{n}_{2}\right)\right\}: .
\end{aligned}
$$

The momentum-dependent coefficient function $f(\vec{q})$ is the same as defined in Eq. 42 and 43 .

\section{Lattice interactions at next-to-leading-order}

The lattice interactions at next-to-leading order were discussed in Ref. [4]. We follow the same formalism here. We start with the corrections to the leading-order "contact" interactions. These NLO interactions are chosen to be point-like rather than smeared operators, and we write the interactions in the same manner as in Ref. [4],

$$
\Delta V=\frac{1}{2} \Delta C: \sum_{\vec{n}} \rho^{a^{\dagger}, a}(\vec{n}) \rho^{a^{\dagger}, a}(\vec{n}):,
$$




$$
\Delta V_{I^{2}}=\frac{1}{2} \Delta C_{I^{2}}: \sum_{\vec{n}, I} \rho_{I}^{a^{\dagger}, a}(\vec{n}) \rho_{I}^{a^{\dagger}, a}(\vec{n}): .
$$

At next-to-leading order there are seven independent contact interactions with two derivatives. The basis we choose is

$$
\begin{gathered}
V_{q^{2}}=-\frac{1}{2} C_{q^{2}}: \sum_{\vec{n}, l} \rho^{a^{\dagger}, a}(\vec{n}) \nabla_{l}^{2} \rho^{a^{\dagger}, a}(\vec{n}): \\
V_{I^{2}, q^{2}}=-\frac{1}{2} C_{I^{2}, q^{2}}: \sum_{\vec{n}, I, l} \rho_{I}^{a^{\dagger}, a}(\vec{n}) \nabla_{l}^{2} \rho_{I}^{a^{\dagger}, a}(\vec{n}): \\
V_{S^{2}, q^{2}}=-\frac{1}{2} C_{S^{2}, q^{2}}: \sum_{\vec{n}, S, l} \rho_{S}^{a^{\dagger}, a}(\vec{n}) \nabla_{l}^{2} \rho_{S}^{a^{\dagger}, a}(\vec{n}):, \\
V_{S^{2}, I^{2}, q^{2}}=-\frac{1}{2} C_{S^{2}, I^{2}, q^{2}}: \sum_{\vec{n}, S, I, l} \rho_{S, I}^{a^{\dagger}, a}(\vec{n}) \nabla_{l}^{2} \rho_{S, I}^{a^{\dagger}, a}(\vec{n}):, \\
V_{(q \cdot S)^{2}}=\frac{1}{2} C_{(q \cdot S)^{2}}: \sum_{\vec{n}} \sum_{S} \Delta_{S} \rho_{S}^{a^{\dagger}, a}(\vec{n}) \sum_{S^{\prime}} \Delta_{S^{\prime}} \rho_{S^{\prime}}^{a^{\dagger}, a}(\vec{n}):, \\
V_{I^{2},(q \cdot S)^{2}}=\frac{1}{2} C_{I^{2},(q \cdot S)^{2}}: \sum_{\vec{n}, I} \sum_{S} \Delta_{S} \rho_{S, I}^{a^{\dagger}, a}(\vec{n}) \sum_{S^{\prime}} \Delta_{S^{\prime}} \rho_{S^{\prime}, I}^{a^{\dagger}, a}(\vec{n}):, \\
V_{(i q \times S) \cdot k}=-\frac{i}{2} C_{(i q \times S) \cdot k} \sum_{\vec{n}, l, S, l^{\prime}} \varepsilon_{l, S, l^{\prime}}\left[\prod_{l}^{a^{\dagger}, a}(\vec{n}) \Delta_{l^{\prime}} \rho_{S}^{a^{\dagger}, a}(\vec{n})+\prod_{l, S}^{a^{\dagger}, a}(\vec{n}) \Delta_{l^{\prime}} \rho^{a^{\dagger}, a}(\vec{n})\right]:
\end{gathered}
$$

These operators are different from those shown in Eq. (6) and allow for a simple projection onto different isospin channels. This will be useful later when restricting to the interactions of neutrons.

The $V_{(i q \times S) \cdot k}$ term corresponds with the continuum interaction

$$
C_{(i q \times S) \cdot k}\left(i \vec{q} \times\left(\vec{\sigma}^{A}+\vec{\sigma}^{B}\right)\right) \cdot \vec{k}
$$

which vanishes unless the total spin is $S=1$. The continuum limit of this interaction is antisymmetric under the exchange of $\vec{q}$ and $\vec{k}$ and is nonzero only for odd parity channels. However the lattice interaction $V_{(i q \times S) \cdot k}$ does not share this exact $t$ - $u$ channel antisymmetry at nonzero lattice spacing. Therefore $V_{(i q \times S) \cdot k}$ has small lattice artifacts for $S=1$ in even parity channels. We remove this defect by including an explicit projection onto total isospin 
$I=1$

$$
\begin{aligned}
V_{(i q \times S) \cdot k}^{I=1}=- & \frac{i}{2} C_{(i q \times S) \cdot k}^{I=1}\left\{\frac{3}{4}: \sum_{\vec{n}, l, S, l^{\prime}} \varepsilon_{l, S, l^{\prime}}\left[\prod_{l}^{a^{\dagger}, a}(\vec{n}) \Delta_{l^{\prime}} \rho_{S}^{a^{\dagger}, a}(\vec{n})+\Pi_{l, S}^{a^{\dagger}, a}(\vec{n}) \Delta_{l^{\prime}} \rho^{a^{\dagger}, a}(\vec{n})\right]:\right. \\
& \left.+\frac{1}{4}: \sum_{\vec{n}, l, S, l^{\prime}, I} \varepsilon_{l, S, l^{\prime}}\left[\prod_{l, I}^{a^{\dagger}, a}(\vec{n}) \Delta_{l^{\prime}} \rho_{S, I}^{a^{\dagger}, a}(\vec{n})+\Pi_{l, S, I}^{a^{\dagger}, a}(\vec{n}) \Delta_{l^{\prime}} \rho_{I}^{a^{\dagger}, a}(\vec{n})\right]:\right\} .
\end{aligned}
$$

This projection completely eliminates lattice artifacts in the $S=1$ even parity channels.

\section{SCATTERING RESULTS FOR $\mathrm{LO}_{3}$ AND $\mathrm{NLO}_{3}$}

We measure phase shifts and mixing angles using the spherical wall method [5]. This consists of imposing a hard spherical wall boundary on the relative separation between the two nucleons at some chosen radius $R_{\text {wall }}$. Scattering phase shifts are determined from the energies of the spherical standing waves, and mixing angles are extracted from projections on to spherical harmonics. At next-to-leading order there are nine unknown operator coefficients: $\Delta C, \Delta C_{I^{2}}, C_{q^{2}}, C_{I^{2}, q^{2}}, C_{S^{2}, q^{2}}, C_{S^{2}, I^{2}, q^{2}}, C_{(q \cdot S)^{2}}, C_{I^{2},(q \cdot S)^{2}}$, and $C_{(i q \times S) \cdot k}^{I=1}$. These nine operator coefficients are fit in the same manner as described in Ref. [4]. For $R_{\text {wall }}=10+\epsilon$ lattice units, where $\epsilon$ is an infinitesimal positive number, we compute energy levels for the eight spherical wall modes listed in Table I. The labelling of these modes is

discussed in Ref. [4]. In addition to these we also consider $Q_{d}$, the quadrupole moment of the deuteron. $Q_{d}$ is a measure of $S-D$ partial wave mixing at low energies and is somewhat easier to compute on the lattice than the $S$ - $D$ mixing angle. For each of the nine observables we compute derivatives with respect to the nine NLO coefficient operators. By inverting the resulting $9 \times 9$ Jacobian matrix, we find values for the NLO coefficients needed to match each of the nine target values using first-order perturbation theory. The results for the operator coefficients are shown in Table II.

With the $\mathrm{NLO}_{3}$ coefficients in hand, we can now calculate lattice phase shifts and mixing angles up to next-to-leading order using the spherical wall method. We consider spherical walls with radii $R_{\text {wall }}=10+\epsilon, 9+\epsilon$, and $8+\epsilon$ lattice units. In order of increasing momentum, the lattice data correspond with the first radial excitation for $R_{\text {wall }}=10+\epsilon, 9+\epsilon$, and $8+\epsilon$; second radial excitation of $R_{\text {wall }}=10+\epsilon, 9+\epsilon$, and $8+\epsilon$; and so on. The $S$-wave phase shifts for $\mathrm{LO}_{3}$ and $\mathrm{NLO}_{3}$ versus center-of-mass momentum $p_{\mathrm{CM}}$ are shown in Fig. 2. The 
TABLE I: Results for $\mathrm{LO}_{3}$ and the physical target values

\begin{tabular}{||c|c|c|c||}
\hline \hline Spherical wave & Free nucleons & $\mathrm{LO}_{3}$ & PWA93 \\
\hline $1^{1} S_{0}(\mathrm{MeV})$ & 0.928 & 0.418 & 0.407 \\
\hline $3^{1} S_{0}(\mathrm{MeV})$ & 8.535 & 6.843 & 6.815 \\
\hline $1^{3} S(D)_{1}(\mathrm{MeV})$ & 0.928 & -2.225 & -2.225 \\
\hline $3^{3} S(D)_{1}(\mathrm{MeV})$ & 8.535 & 5.430 & 5.675 \\
\hline $2^{1} P_{1}(\mathrm{MeV})$ & 5.691 & 5.755 & 5.782 \\
\hline $2^{3} P(F)_{0}(\mathrm{MeV})$ & 5.691 & 5.569 & 5.584 \\
\hline $2^{3} P(F)_{1}(\mathrm{MeV})$ & 5.691 & 5.754 & 5.753 \\
\hline $2^{3} P(F)_{2}(\mathrm{MeV})$ & 5.691 & 5.684 & 5.669 \\
\hline$Q_{d}\left(\mathrm{fm}^{2}\right)$ & N/A & 0.276 & 0.286 \\
\hline \hline
\end{tabular}

TABLE II: Results for $\mathrm{NLO}_{3}$ operator coefficients

\begin{tabular}{||c|c||}
\hline \hline Coefficient & $\mathrm{NLO}_{3}$ \\
\hline$\Delta C\left(\mathrm{MeV}^{-2}\right)$ & $-1.02 \times 10^{-5}$ \\
\hline$\Delta C_{I^{2}}\left(\mathrm{MeV}^{-2}\right)$ & $1.03 \times 10^{-5}$ \\
\hline$C_{q^{2}}\left(\mathrm{MeV}^{-4}\right)$ & $2.39 \times 10^{-10}$ \\
\hline$C_{I^{2}, q^{2}}\left(\mathrm{MeV}^{-4}\right)$ & $-4.80 \times 10^{-11}$ \\
\hline$C_{S^{2}, q^{2}}\left(\mathrm{MeV}^{-4}\right)$ & $1.67 \times 10^{-10}$ \\
\hline$C_{S^{2}, I^{2}, q^{2}}\left(\mathrm{MeV}^{-4}\right)$ & $-1.03 \times 10^{-10}$ \\
\hline$C_{(q \cdot S)^{2}}\left(\mathrm{MeV}^{-4}\right)$ & $-1.43 \times 10^{-10}$ \\
\hline$C_{I^{2},(q \cdot S)^{2}}\left(\mathrm{MeV}^{-4}\right)$ & $1.80 \times 10^{-10}$ \\
\hline$C_{(i q \times S) \cdot k}^{I=1}\left(\mathrm{MeV}^{-4}\right)$ & $1.60 \times 10^{-10}$ \\
\hline \hline
\end{tabular}

$\mathrm{NLO}_{3}$ results are in good agreement with partial wave results from Ref. [30].

We plot the $S$-D mixing parameter $\varepsilon_{1}$ in the Stapp parameterization [31] in Fig. [3]. The pairs of points connected by dotted lines indicate pairs of coupled solutions in the spherical wall formalism. While there are some deviations from the partial wave data from Ref. [30], the discrepancy is consistent with effects produced by higher-order interactions. As expected the $S$-wave results for $\mathrm{LO}_{3}$ are identical with $\mathrm{LO}_{2}$ results in Ref. [5]. In fact they agree in all 

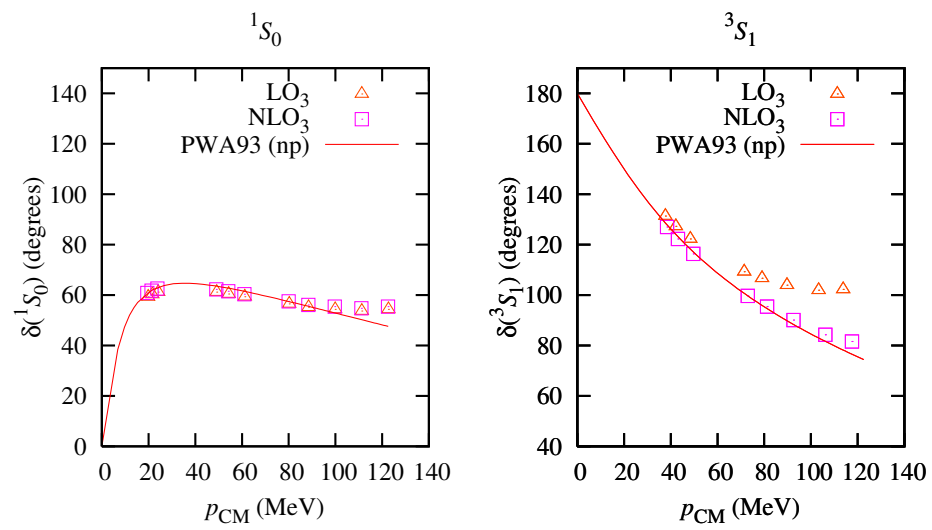

FIG. 2: $S$-wave phase shifts versus center-of-mass momentum for $\mathrm{LO}_{3}$ and $\mathrm{NLO}_{3}$.

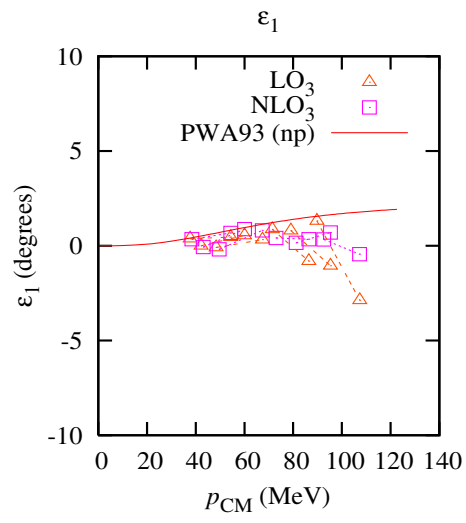

FIG. 3: $\varepsilon_{1}$ mixing angle versus center-of-mass momentum for $\mathrm{LO}_{3}$ and $\mathrm{NLO}_{3}$.

even- $L$ partial wave channels. Results for $\mathrm{NLO}_{3}$ and $\mathrm{NLO}_{2}$ are also close though not exactly the same. There are very small differences between the two due to NLO interactions which are not completely separable into $S$-wave and $P$-wave terms at nonzero lattice spacing.

The $P$-wave phase shifts are shown in Fig. 4. We see that the $\mathrm{NLO}_{3}$ results match the partial wave data quite accurately. Just as $\mathrm{LO}_{3}$ and $\mathrm{LO}_{2}$ agree in all even- $L$ partial wave channels, $\mathrm{LO}_{3}$ and $\mathrm{LO}_{1}$ agree in all odd- $L$ partial wave channels. Results for $\mathrm{NLO}_{3}$ and $\mathrm{NLO}_{1}$ are nearly identical, with only small differences due to the numerical fitting of NLO coefficients on the lattice. 

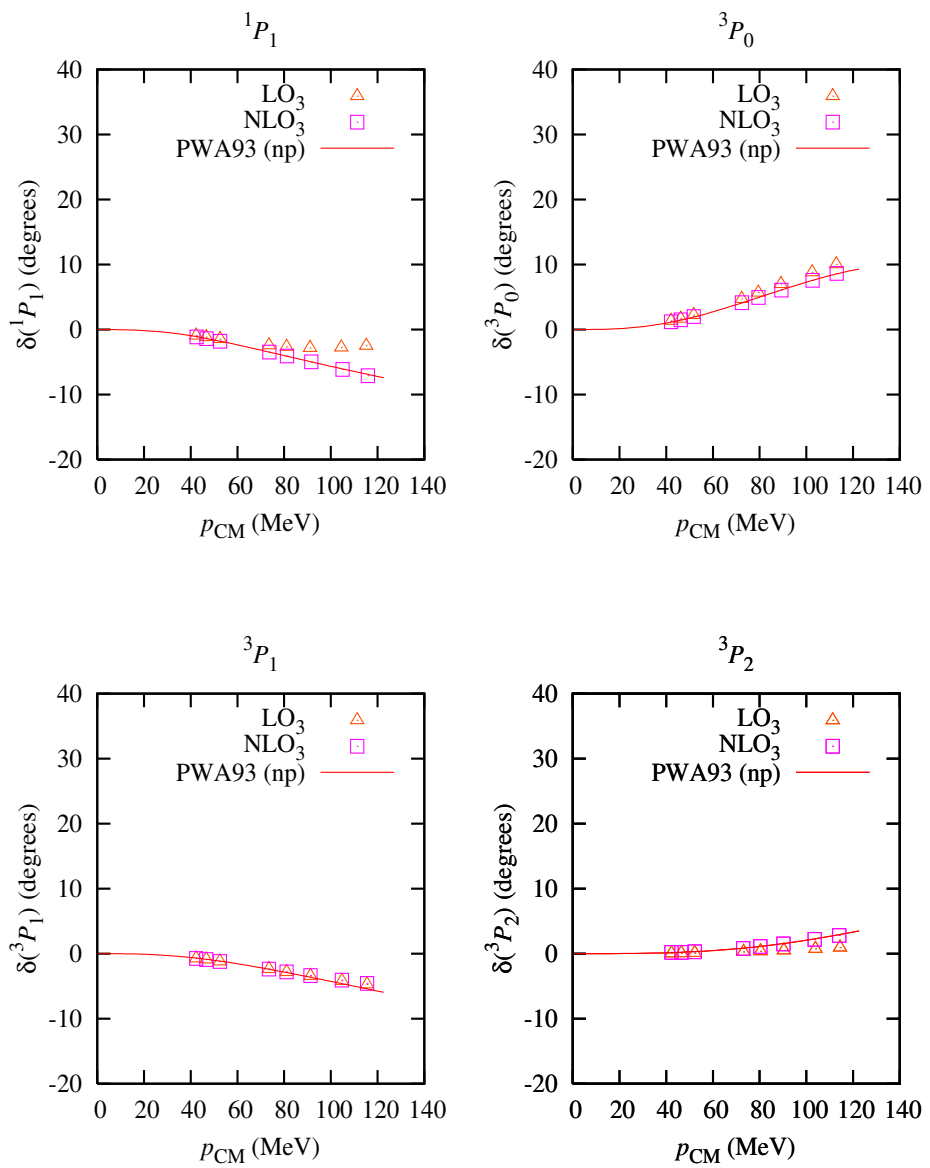

FIG. 4: $P$-wave phase shifts versus center-of-mass momentum for $\mathrm{LO}_{3}$ and $\mathrm{NLO}_{3}$.

\section{AUXILIARY-FIELD FORMALISM FOR NEUTRON MATTER}

So far we have been discussing general systems of low-energy nucleons with both protons and neutrons. For computational efficiency we now specialize to the case where all nucleons are neutrons. In this case all nucleon-nucleon interactions are in the isospin-triplet channel. In the leading-order transfer matrix $M_{\mathrm{LO}_{3}}$ we can drop the spin-triplet/isospin-singlet term involving $V_{S=1, I=0}(\vec{q})$ and make the simplifying replacements,

$$
\begin{gathered}
V_{S=0, I=1}(\vec{q}) \rightarrow \frac{1}{8}: \rho^{a^{\dagger}, a}(\vec{q}) \rho^{a^{\dagger}, a}(-\vec{q}):-\frac{1}{8}: \sum_{S} \rho_{S}^{a^{\dagger}, a}(\vec{q}) \rho_{S}^{a^{\dagger}, a}(-\vec{q}): \\
\sum_{S_{1}, S_{2}, I} \sum_{\vec{n}_{1}, \vec{n}_{2}} G_{S_{1} S_{2}}\left(\vec{n}_{1}-\vec{n}_{2}\right) \rho_{S_{1}, I}^{a^{\dagger}, a}\left(\vec{n}_{1}\right) \rho_{S_{2}, I}^{a^{\dagger}, a}\left(\vec{n}_{2}\right) \rightarrow \sum_{S_{1}, S_{2}} \sum_{\vec{n}_{1}, \vec{n}_{2}} G_{S_{1} S_{2}}\left(\vec{n}_{1}-\vec{n}_{2}\right) \rho_{S_{1}}^{a^{\dagger}, a}\left(\vec{n}_{1}\right) \rho_{S_{2}}^{a^{\dagger}, a}\left(\vec{n}_{2}\right) .
\end{gathered}
$$


These modifications do not affect the interactions between neutrons and yields the simplified transfer matrix,

$$
\begin{aligned}
M_{\mathrm{LO}_{3}} \rightarrow & : \exp \left\{-H_{\text {free }} \alpha_{t}-\frac{C_{S=0, I=1} \alpha_{t}}{8 L^{3}} \sum_{\vec{q}} f(\vec{q})\left[\rho^{a^{\dagger}, a}(\vec{q}) \rho^{a^{\dagger}, a}(-\vec{q})-\sum_{S} \rho_{S}^{a^{\dagger}, a}(\vec{q}) \rho_{S}^{a^{\dagger}, a}(-\vec{q})\right]\right. \\
& \left.+\frac{g_{A}^{2} \alpha_{t}^{2}}{8 f_{\pi}^{2} q_{\pi}} \sum_{S_{1}, S_{2}} \sum_{\vec{n}_{1}, \vec{n}_{2}} G_{S_{1} S_{2}}\left(\vec{n}_{1}-\vec{n}_{2}\right) \rho_{S_{1}}^{a^{\dagger}, a}\left(\vec{n}_{1}\right) \rho_{S_{2}}^{a^{\dagger}, a}\left(\vec{n}_{2}\right)\right\}:
\end{aligned}
$$

At next-to-leading order the simplified neutron transfer matrix is

$$
\begin{aligned}
M_{\mathrm{NLO}_{3}} & \rightarrow: \exp \left\{-H_{\mathrm{free}} \alpha_{t}-\frac{C_{S=0, I=1} \alpha_{t}}{8 L^{3}} \sum_{\vec{q}} f(\vec{q})\left[\rho^{a^{\dagger}, a}(\vec{q}) \rho^{a^{\dagger}, a}(-\vec{q})-\sum_{S} \rho_{S}^{a^{\dagger}, a}(\vec{q}) \rho_{S}^{a^{\dagger}, a}(-\vec{q})\right]\right. \\
& -\alpha_{t}\left[\frac{\Delta C^{I=1}}{\Delta C} \Delta V+\frac{C_{q^{2}}^{I=1}}{C_{q^{2}}} V_{q^{2}}+\frac{C_{S^{2}, q^{2}}^{I=1}}{C_{S^{2}, q^{2}}} V_{S^{2}, q^{2}}+\frac{C_{(q \cdot S)^{2}}^{I=1}}{C_{(q \cdot S)^{2}}} V_{(q \cdot S)^{2}}+V_{(i q \times S) \cdot k}^{I=1}\right] \\
& \left.+\frac{g_{A}^{2} \alpha_{t}^{2}}{8 f_{\pi}^{2} q_{\pi}} \sum_{S_{1}, S_{2}, \vec{n}_{1}, \vec{n}_{2}} G_{S_{1} S_{2}}\left(\vec{n}_{1}-\vec{n}_{2}\right) \rho_{S_{1}}^{a^{\dagger}, a}\left(\vec{n}_{1}\right) \rho_{S_{2}}^{a^{\dagger}, a}\left(\vec{n}_{2}\right)\right\}:
\end{aligned}
$$

In the following we use these simplified forms for the leading-order and next-to-leading-order transfer matrices.

The transfer matrices in Eq. (62) and (63) can be rewritten in terms of one-body interactions with auxiliary fields. The exact equivalence between lattice formalisms with and without auxiliary fields is detailed in [1, 32, 33]. We summarize the results here.

In neutron-neutron scattering only the neutral pion contributes to one-pion exchange. We have been writing the rescaled neutral pion field as $\pi_{3}^{\prime}$, but now we drop the subscript "3" and simply write $\pi^{\prime}$. Let $M^{\left(n_{t}\right)}\left(\pi^{\prime}, s, s_{S}\right)$ be the leading-order auxiliary-field transfer matrix at time step $n_{t}$,

$$
\begin{aligned}
M^{\left(n_{t}\right)}\left(\pi^{\prime}, s, s_{S}\right)=: \exp \{ & -H_{\text {free }} \alpha_{t}+\frac{g_{A} \alpha_{t}}{2 f_{\pi} \sqrt{q_{\pi}}} \sum_{\vec{n}, S} \Delta_{S} \pi^{\prime}\left(\vec{n}, n_{t}\right) \rho_{S}^{a^{\dagger}, a}(\vec{n}) \\
& +\frac{1}{2} \sqrt{-C_{S=0, I=1} \alpha_{t}} \sum_{\vec{n}} s\left(\vec{n}, n_{t}\right) \rho^{a^{\dagger}, a}(\vec{n}) \\
& \left.+\frac{i}{2} \sqrt{-C_{S=0, I=1} \alpha_{t}} \sum_{\vec{n}, S} s_{S}\left(\vec{n}, n_{t}\right) \rho_{S}^{a^{\dagger}, a}(\vec{n})\right\}: .
\end{aligned}
$$


We can write $M_{\mathrm{LO}_{3}}$ as the normalized integral

$$
M_{\mathrm{LO}_{3}}=\frac{\int D \pi^{\prime} D s D s_{S} e^{-S_{\pi \pi}^{\left(n_{t}\right)}-S_{s s}^{\left(n_{t}\right)}} M^{\left(n_{t}\right)}\left(\pi^{\prime}, s, s_{S}\right)}{\int D \pi^{\prime} D s D s_{S} e^{-S_{\pi \pi}^{\left(n_{t}\right)}-S_{s s}^{\left(n_{t}\right)}}},
$$

where $S_{\pi \pi}^{\left(n_{t}\right)}$ is the piece of the instantaneous pion action in Eq. (28) containing the neutral pion field at time step $n_{t}$,

$$
S_{\pi \pi}^{\left(n_{t}\right)}\left(\pi^{\prime}\right)=\frac{1}{2} \sum_{\vec{n}} \pi^{\prime}\left(\vec{n}, n_{t}\right) \pi^{\prime}\left(\vec{n}, n_{t}\right)-\frac{\alpha_{t}}{q_{\pi}} \sum_{\vec{n}, l} \pi^{\prime}\left(\vec{n}, n_{t}\right) \pi^{\prime}\left(\vec{n}+\hat{l}, n_{t}\right),
$$

and $S_{s s}^{\left(n_{t}\right)}$ is the auxiliary-field action at time step $n_{t}$,

$$
S_{s s}^{\left(n_{t}\right)}\left(s, s_{S}\right)=\frac{1}{2} \sum_{\vec{n}, \vec{n}^{\prime}} s\left(\vec{n}, n_{t}\right) f^{-1}\left(\vec{n}-\vec{n}^{\prime}\right) s\left(\vec{n}^{\prime}, n_{t}\right)+\frac{1}{2} \sum_{\vec{n}, \vec{n}^{\prime}, S} s_{S}\left(\vec{n}, n_{t}\right) f^{-1}\left(\vec{n}-\vec{n}^{\prime}\right) s_{S}\left(\vec{n}^{\prime}, n_{t}\right),
$$

with

$$
f^{-1}\left(\vec{n}-\vec{n}^{\prime}\right)=\frac{1}{L^{3}} \sum_{\vec{q}} \frac{1}{f(\vec{q})} e^{-i \vec{q} \cdot\left(\vec{n}-\vec{n}^{\prime}\right)} .
$$

The NLO interactions require some additional auxiliary fields. Let

$$
\begin{aligned}
U^{\left(n_{t}\right)}(\varepsilon) & =\sum_{\vec{n}} \varepsilon_{\rho}\left(\vec{n}, n_{t}\right) \rho^{a^{\dagger}, a}(\vec{n})+\sum_{\vec{n}, S} \varepsilon_{\rho_{S}}\left(\vec{n}, n_{t}\right) \rho_{S}^{a^{\dagger}, a}(\vec{n})+\sum_{\vec{n}, S} \varepsilon_{\Delta_{S} \rho}\left(\vec{n}, n_{t}\right) \Delta_{S} \rho^{a^{\dagger}, a}(\vec{n}) \\
& +\sum_{\vec{n}, S, S^{\prime}} \varepsilon_{\Delta_{S} \rho_{S^{\prime}}}\left(\vec{n}, n_{t}\right) \Delta_{S} \rho_{S^{\prime}}^{a^{\dagger}, a}(\vec{n})+\sum_{\vec{n}, l} \varepsilon_{\nabla_{l}^{2} \rho}\left(\vec{n}, n_{t}\right) \nabla_{l}^{2} \rho^{a^{\dagger}, a}(\vec{n}) \\
& +\sum_{\vec{n}, l, S} \varepsilon_{\nabla_{l}^{2} \rho_{S}}\left(\vec{n}, n_{t}\right) \nabla_{l}^{2} \rho_{S}^{a^{\dagger}, a}(\vec{n})+\sum_{\vec{n}, l} \varepsilon_{\Pi_{l}}\left(\vec{n}, n_{t}\right) \Pi_{l}^{a^{\dagger}, a}(\vec{n})+\sum_{\vec{n}, l, S} \varepsilon_{\Pi_{l, S}}\left(\vec{n}, n_{t}\right) \Pi_{l, S}^{a^{\dagger}, a}(\vec{n}) .
\end{aligned}
$$

With these extra fields and linear functional $U^{\left(n_{t}\right)}(\varepsilon)$ we define

$$
\begin{aligned}
M^{\left(n_{t}\right)}\left(\pi^{\prime}, s, s_{S}, \varepsilon\right)=: \exp \{ & -H_{\text {free }} \alpha_{t}+\frac{g_{A} \alpha_{t}}{2 f_{\pi} \sqrt{q_{\pi}}} \sum_{\vec{n}, S} \Delta_{S} \pi^{\prime}\left(\vec{n}, n_{t}\right) \rho_{S}^{a^{\dagger}, a}(\vec{n}) \\
& +\frac{1}{2} \sqrt{-C_{S=0, I=1} \alpha_{t}} \sum_{\vec{n}} s\left(\vec{n}, n_{t}\right) \rho^{a^{\dagger}, a}(\vec{n}) \\
& \left.+\frac{i}{2} \sqrt{-C_{S=0, I=1} \alpha_{t}} \sum_{\vec{n}, S} s_{S}\left(\vec{n}, n_{t}\right) \rho_{S}^{a^{\dagger}, a}(\vec{n})+\sqrt{\alpha_{t}} U^{\left(n_{t}\right)}(\varepsilon)\right\}: .
\end{aligned}
$$

We also define the normalized integral,

$$
M^{\left(n_{t}\right)}(\varepsilon)=\frac{\int D \pi^{\prime} D s D s_{S} e^{-S_{\pi \pi}^{\left(n_{t}\right)}-S_{s s}^{\left(n_{t}\right)}} M^{\left(n_{t}\right)}\left(\pi^{\prime}, s, s_{S}, \varepsilon\right)}{\int D \pi^{\prime} D s D s_{S} e^{-S_{\pi \pi}^{\left(n_{t}\right)}-S_{s s}^{\left(n_{t}\right)}}} .
$$


When all $\varepsilon$ fields are set to zero we recover $M_{\mathrm{LO}_{3}}$,

$$
M^{\left(n_{t}\right)}(0)=M_{\mathrm{LO}_{3}}
$$

To first order the NLO interactions in $M_{\mathrm{NLO}_{3}}$ can be written as a sum of bilinear derivatives of $M^{\left(n_{t}\right)}(\varepsilon)$ with respect to the $\varepsilon$ fields at $\varepsilon=0$,

$$
\begin{aligned}
M_{\mathrm{NLO}_{3}} & =M_{\mathrm{LO}_{3}} \\
& -\left.\frac{1}{2} \Delta C^{I=1} \sum_{\vec{n}} \frac{\delta}{\delta \varepsilon_{\rho}\left(\vec{n}, n_{t}\right)} \frac{\delta}{\delta \varepsilon_{\rho}\left(\vec{n}, n_{t}\right)} M^{\left(n_{t}\right)}(\varepsilon)\right|_{\varepsilon=0} \\
& +\left.\frac{1}{2} C_{q^{2}}^{I=1} \sum_{\vec{n}} \frac{\delta}{\delta \varepsilon_{\rho}\left(\vec{n}, n_{t}\right)} \frac{\delta}{\delta \varepsilon_{\nabla_{l}^{2} \rho}\left(\vec{n}, n_{t}\right)} M^{\left(n_{t}\right)}(\varepsilon)\right|_{\varepsilon=0}+\cdots
\end{aligned}
$$

\section{EUCLIDEAN-TIME PROJECTION MONTE CARLO}

We extract the properties of the ground state using Euclidean-time projection. We briefly summarize the calculation in continuous-time notation before describing the transfer matrix calculation at nonzero temporal lattice spacing. Let $\left|\Psi^{\text {free }}\right\rangle$ be a Slater determinant of freeparticle standing waves in a periodic cube for $N$ neutrons. Let $H_{\mathrm{LO}_{3}}$ be the Hamiltonian at leading order and $H_{\mathrm{NLO}_{3}}$ be the Hamiltonian at next-to-leading order. Let $H_{\mathrm{SU}(2) \not x}$ be the same as $H_{\mathrm{LO}_{3}}$, but with one-pion exchange turned off by setting $g_{A}$ to zero. As the notation suggests, $H_{\mathrm{SU}(2) \not x}$ is invariant under an exact $\mathrm{SU}(2)$ intrinsic-spin symmetry. We define a trial wavefunction

$$
\left|\Psi\left(t^{\prime}\right)\right\rangle=\exp \left(-H_{\mathrm{SU}(2) \not t^{\prime}} t^{\prime}\left|\Psi^{\text {free }}\right\rangle .\right.
$$

The operator $\exp \left(-H_{\mathrm{SU}(2) \not t^{\prime}}\right)$ acts as an approximate low-energy filter. In the auxiliaryfield Monte Carlo calculation this part of the Euclidean-time propagation is positive definite for any even number of neutrons invariant under the $\mathrm{SU}(2)$ intrinsic-spin symmetry [34, 35, 36]. With this trial wavefunction we define the amplitude,

$$
Z(t)=\left\langle\Psi\left(t^{\prime}\right)\left|\exp \left(-H_{\mathrm{LO}_{3}} t\right)\right| \Psi\left(t^{\prime}\right)\right\rangle
$$

as well as the transient energy at Euclidean time $t$,

$$
E_{\mathrm{LO}_{3}}(t)=-\frac{\partial}{\partial t}[\ln Z(t)]
$$


In the limit of large $t$,

$$
\lim _{t \rightarrow \infty} E_{\mathrm{LO}_{3}}(t)=E_{0, \mathrm{LO}_{3}},
$$

where $E_{0, \mathrm{LO}}$ is the energy of the lowest eigenstate $\left|\Psi_{0}\right\rangle$ of $H_{\mathrm{LO}_{3}}$ with nonzero inner product with $\left|\Psi\left(t^{\prime}\right)\right\rangle$.

To compute the expectation value of some general operator $O$ we define

$$
Z_{O}(t)=\left\langle\Psi\left(t^{\prime}\right)\left|\exp \left(-H_{\mathrm{LO}_{3}} t / 2\right) O \exp \left(-H_{\mathrm{LO}_{3}} t / 2\right)\right| \Psi\left(t^{\prime}\right)\right\rangle
$$

The expectation value of $O$ for $\left|\Psi_{0}\right\rangle$ is given by the large $t$ limit,

$$
\lim _{t \rightarrow \infty} \frac{Z_{O}(t)}{Z(t)}=\left\langle\Psi_{0}|O| \Psi_{0}\right\rangle
$$

Corrections to the energy at next-to-leading order can be computed using $O=H_{\mathrm{NLO}_{3}}-H_{\mathrm{LO}_{3}}$. In that case

$$
\lim _{t \rightarrow \infty} \frac{Z_{O}(t)}{Z(t)}=E_{0, \mathrm{NLO}_{3}}-E_{0, \mathrm{LO}_{3}}
$$

where $E_{0, \mathrm{NLO}_{3}}$ is the ground state energy at next-to-leading order.

On the lattice we construct $\left|\Psi\left(t^{\prime}\right)\right\rangle$ using

$$
\left|\Psi\left(t^{\prime}\right)\right\rangle=\left(M_{\mathrm{SU}(2) \not x^{\prime}}\right)^{L_{t_{o}}}\left|\Psi^{\text {free }}\right\rangle
$$

where $t^{\prime}=L_{t_{o}} \alpha_{t}$ and $L_{t_{o}}$ is the number of "outer" time steps. The amplitude $Z(t)$ is defined as

$$
Z(t)=\left\langle\Psi\left(t^{\prime}\right)\left|\left(M_{\mathrm{LO}_{3}}\right)^{L_{t_{i}}}\right| \Psi\left(t^{\prime}\right)\right\rangle
$$

where $t=L_{t_{i}} \alpha_{t}$ and $L_{t_{i}}$ is the number of "inner" time steps. The transient energy

$$
E_{\mathrm{LO}_{3}}\left(t+\alpha_{t} / 2\right)
$$

is given by the ratio of the amplitudes for $t$ and $t+\alpha_{t}$,

$$
e^{-E_{\mathrm{LO}_{3}}\left(t+\alpha_{t} / 2\right) \cdot \alpha_{t}}=\frac{Z\left(t+\alpha_{t}\right)}{Z(t)} .
$$

The ground state energy $E_{0, \mathrm{LO}_{3}}$ equals the asymptotic limit,

$$
E_{0, \mathrm{LO}_{3}}=\lim _{t \rightarrow \infty} E_{\mathrm{LO}_{3}}\left(t+\alpha_{t} / 2\right)
$$

We calculate these Euclidean-time projection amplitudes using auxiliary fields. For a given configuration of auxiliary and pion fields, the contribution to the amplitude $Z(t)$ is 
proportional to the determinant of an $N \times N$ matrix of one-body amplitudes, where $N$ is the number of neutrons. Integrations over auxiliary and pion field configurations are computed using hybrid Monte Carlo. Details of the method can be found in Ref. [1, 32, 33, 37].

For the ground state energy at next-to-leading order we compute expectation values of $M_{\mathrm{NLO}_{3}}$ and $M_{\mathrm{LO}_{3}}$ inserted in the middle of a string of $M_{\mathrm{LO}_{3}}$ transfer matrices,

$$
\begin{aligned}
Z_{M_{\mathrm{NLO}_{3}}}(t) & =\left\langle\Psi\left(t^{\prime}\right)\left|\left(M_{\mathrm{LO}_{3}}\right)^{L_{t_{i}} / 2} M_{\mathrm{NLO}_{3}}\left(M_{\mathrm{LO}_{3}}\right)^{L_{t_{i}} / 2}\right| \Psi\left(t^{\prime}\right)\right\rangle, \\
Z_{M_{\mathrm{LO}_{3}}}(t) & =\left\langle\Psi\left(t^{\prime}\right)\left|\left(M_{\mathrm{LO}_{3}}\right)^{L_{t_{i}} / 2} M_{\mathrm{LO}_{3}}\left(M_{\mathrm{LO}_{3}}\right)^{L_{t_{i}} / 2}\right| \Psi\left(t^{\prime}\right)\right\rangle .
\end{aligned}
$$

From the ratio of amplitudes,

$$
\frac{Z_{M_{\mathrm{NLO}_{3}}}(t)}{Z_{M_{\mathrm{LO}}}(t)}=1-\Delta E_{\mathrm{NLO}_{3}}(t) \alpha_{t}+\cdots,
$$

we define the transient NLO energy correction $\Delta E_{\mathrm{NLO}_{3}}(t)$. The ellipsis denotes terms which are beyond first order in the NLO coefficients. The NLO ground state energy $E_{0, \mathrm{NLO}_{3}}$ is calculated using

$$
E_{0, \mathrm{NLO}_{3}}=E_{0, \mathrm{LO}_{3}}+\lim _{t \rightarrow \infty} \Delta E_{0, \mathrm{NLO}_{3}}(t)
$$

\section{PRECISION TESTS OF MONTE CARLO SIMULATIONS}

We use the two-neutron system to test the auxiliary-field Monte Carlo simulations. The same observables are calculated using both auxiliary-field Monte Carlo and the exact transfer matrix without auxiliary fields. We choose a small system so that stochastic errors are small enough to expose disagreement at the $0.1 \%-1 \%$ level. We choose the spatial length of lattice to be $L=4$ and set the outer time steps $L_{t_{o}}=2$ and inner time steps $L_{t_{i}}=2$. With 16 processors we generate a total of about $8 \times 10^{5}$ hybrid Monte Carlo trajectories. Each processor runs completely independent trajectories, and we compute averages and stochastic errors by comparing the results of all processors.

For the first test we choose $\left|\Psi^{\text {free }}\right\rangle$ to be a spin-singlet state built from the Slater determinant of standing waves $\left|\psi_{1}\right\rangle$ and $\left|\psi_{2}\right\rangle$ with

$$
\left\langle 0\left|a_{i, j}(\vec{n})\right| \psi_{1}\right\rangle \propto \delta_{i, 0} \delta_{j, 1}, \quad\left\langle 0\left|a_{i, j}(\vec{n})\right| \psi_{2}\right\rangle \propto \delta_{i, 1} \delta_{j, 1}
$$

For the second test we choose a spin-triplet state with standing waves

$$
\left\langle 0\left|a_{i, j}(\vec{n})\right| \psi_{1}\right\rangle \propto \delta_{i, 0} \delta_{j, 1} \cos \frac{2 \pi n_{1}}{L}, \quad\left\langle 0\left|a_{i, j}(\vec{n})\right| \psi_{2}\right\rangle \propto \delta_{i, 0} \delta_{j, 1} \sin \frac{2 \pi n_{1}}{L}
$$


TABLE III: Monte Carlo results versus exact transfer matrix calculations for the two-neutron spin singlet $S=0$ and spin triplet $S=1$.

\begin{tabular}{|c|c|c|c|c|}
\hline & $S=0(\mathrm{MC})$ & $S=0$ (exact) & $S=1(\mathrm{MC})$ & $S=1$ (exact) \\
\hline$E_{\mathrm{LO}_{3}}\left(t+\alpha_{t} / 2\right)[\mathrm{MeV}]$ & $-2.90(2)$ & -2.9112 & $28.3(2)$ & 28.3658 \\
\hline$\frac{\partial\left(\Delta E_{\mathrm{NLO}_{3}}(t)\right)}{\partial\left(\Delta C^{I=1}\right)}\left[10^{4} \mathrm{MeV}^{3}\right]$ & $4.751(5)$ & 4.7487 & $0.0003(7)$ & 0 \\
\hline$\frac{\partial\left(\Delta E_{\mathrm{NLO}_{3}}(t)\right)}{\partial\left(C_{q^{2}}^{I=1}\right)}\left[10^{9} \mathrm{MeV}^{5}\right]$ & $1.580(2)$ & 1.5789 & $-1.025(4)$ & -1.0264 \\
\hline$\frac{\partial\left(\Delta E_{\mathrm{NLO}_{3}}(t)\right)}{\partial\left(C_{S^{2}, q^{2}}^{I=1}\right)}\left[10^{9} \mathrm{MeV}^{5}\right]$ & $-4.741(6)$ & -4.7366 & $-1.023(6)$ & -1.0264 \\
\hline$\frac{\partial\left(\Delta E_{\mathrm{NLO}_{3}}(t)\right)}{\partial\left(C_{(q \cdot S)^{2}}^{I=1}\right)}\left[10^{8} \mathrm{MeV}^{5}\right]$ & $-5.788(8)$ & -5.7818 & $2.51(2)$ & 2.5533 \\
\hline$\frac{\partial\left(\Delta E_{\mathrm{NLO}_{3}}(t)\right)}{\partial\left(C_{(i q \times S) \cdot k}^{I=1}\right)}\left[10^{7} \mathrm{MeV}^{5}\right]$ & $0.018(13)$ & 0 & $3.27(16)$ & 3.4534 \\
\hline$\Delta E_{\mathrm{NLO}_{3}}(t)[\mathrm{MeV}]$ & $-0.01655(8)$ & -0.016440 & $-0.2455(9)$ & -0.24559 \\
\hline
\end{tabular}

Comparisons between Monte Carlo results (MC) and exact transfer matrix calculations (exact) are shown in Table III. The numbers in parentheses are the estimated stochastic errors. The agreement between Monte Carlo results and exact transfer calculations is consistent with the estimated stochastic errors.

\section{RESULTS}

We simulate the ground state for $N=8,12,16$ neutrons on periodic cube lattices. For $N=8$ we consider cube lengths $L=4,5,6,7$ lattice units. For $N=12$, we use $L=5,6,7$, and for $N=16$ we use $L=6,7$. For each value of $N$ and $L$ we fix $L_{t_{o}}$ at either 8 or 10 and vary $L_{t_{i}}$ from 2 up to 12 . For $\left|\Psi^{\text {free }}\right\rangle$ we take the Slater determinant formed by standing waves

$$
\left\langle 0\left|a_{i, j}(\vec{n})\right| \psi_{2 k+1}\right\rangle \propto \delta_{i, 0} \delta_{j, 1} f_{k}(\vec{n}), \quad\left\langle 0\left|a_{i, j}(\vec{n})\right| \psi_{2 k+2}\right\rangle \propto \delta_{i, 1} \delta_{j, 1} f_{k}(\vec{n}),
$$

where

$$
\begin{gathered}
f_{0}(\vec{n})=1, \quad f_{1}(\vec{n})=\cos \frac{2 \pi n_{3}}{L}, \quad f_{2}(\vec{n})=\sin \frac{2 \pi n_{3}}{L}, \quad f_{3}(\vec{n})=\cos \frac{2 \pi n_{1}}{L}, \\
f_{4}(\vec{n})=\sin \frac{2 \pi n_{1}}{L}, \quad f_{5}(\vec{n})=\cos \frac{2 \pi n_{2}}{L}, \quad f_{6}(\vec{n})=\sin \frac{2 \pi n_{2}}{L}, \quad f_{7}(\vec{n})=\cos \frac{2 \pi\left(n_{1}+n_{2}\right)}{L} .
\end{gathered}
$$

For $N=8$ the values of $k$ span the range $0 \leq k \leq 3$. For $N=12,0 \leq k \leq 5$, and for $N=16,0 \leq k \leq 7$. For each value of $L_{t_{i}}$ a total of about $5 \times 10^{6}$ hybrid Monte 
Carlo trajectories are generated by 2048 processors, each running completely independent trajectories. Averages and stochastic errors are computed by comparing the results of all processors.

Let $E_{0}^{\text {free }}$ be the energy of the ground state for noninteracting neutrons. In Fig. 5 we show the dimensionless ratios

$$
\frac{E_{\mathrm{LO}_{3}}(t)}{E_{0}^{\text {free }}}, \frac{\Delta E_{\mathrm{NLO}_{3}}(t)}{E_{0}^{\text {free }}}, \frac{E_{\mathrm{LO}_{3}}(t)+\Delta E_{\mathrm{NLO}_{3}}(t)}{E_{0}^{\text {free }}},
$$

versus Euclidean time $t$. These are labelled using the shorthand $\mathrm{LO}_{3}, \Delta \mathrm{NLO}_{3}$, and $\mathrm{NLO}_{3}$ respectively. In addition to the Monte Carlo data we plot the asymptotic expressions,

$$
\begin{gathered}
\frac{E_{\mathrm{LO}_{3}}(t)}{E_{0}^{\text {free }}} \approx \frac{E_{0, \mathrm{LO}_{3}}}{E_{0}^{\text {free }}}+A e^{-\delta E \cdot t}, \\
\frac{\Delta E_{\mathrm{NLO}_{3}}(t)}{E_{0}^{\text {free }}} \approx \frac{E_{0, \mathrm{NLO}_{3}}-E_{0, \mathrm{LO}}}{E_{0}^{\text {free }}}+B e^{-\delta E \cdot t / 2} . \\
\frac{E_{\mathrm{LO}_{3}}(t)+\Delta E_{\mathrm{NLO}_{3}}(t)}{E_{0}^{\text {free }}} \approx \frac{E_{0, \mathrm{NLO}}}{E_{0}^{\text {free }}}+A e^{-\delta E \cdot t}+B e^{-\delta E \cdot t / 2} .
\end{gathered}
$$

The unknown coefficients $A$ and $B$, energy gap $\delta E$, and ground state energies $E_{0, \mathrm{LO}_{3}}$ and $E_{0, \mathrm{NLO}_{3}}$ are determined by least squares fitting. The $e^{-\delta E \cdot t}$ dependence in Eq. (96) comes from the contribution of low-energy excitations with energy gap $\delta E$ above the ground state. The $e^{-\delta E \cdot t / 2}$ dependence in Eq. (97) comes from the matrix element of $M_{\mathrm{NLO}_{3}}$ between the ground state and low-energy excitations at energy gap $\delta E$.

The results of the asymptotic fits for $E_{0, \mathrm{LO}_{3}} / E_{0}^{\text {free }}$ and $E_{0, \mathrm{NLO}_{3}} / E_{0}^{\text {free }}$ are shown in Table IV] On average the $\chi^{2}$ per degree of freedom for the fits is around 1. The error estimates for $E_{0, \mathrm{LO}_{3}} / E_{0}^{\text {free }}$ and $E_{0, \mathrm{NLO}_{3}} / E_{0}^{\text {free }}$ are calculated by explicit simulation. We introduce Gaussianrandom noise scaled by the error bars of each data point. The fit is repeated many times with the random noise included to estimate the one standard-deviation spread in the fit parameters. In Table IV the Fermi momentum $k_{F}$ for each neutron spin is calculated from the density of neutrons in the periodic cube,

$$
k_{F}=\frac{1}{L}\left(3 \pi^{2} N\right)^{1 / 3}
$$




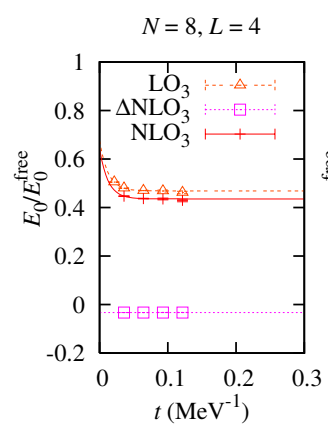

(a)

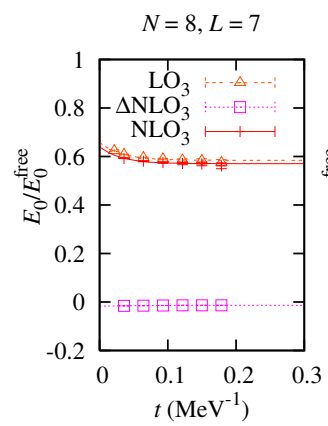

(d)

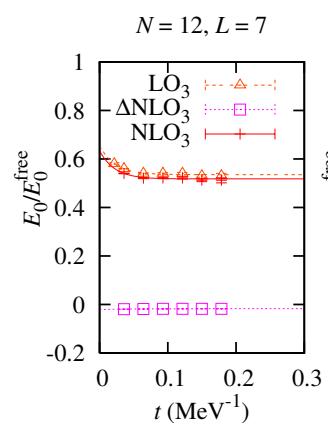

(g)

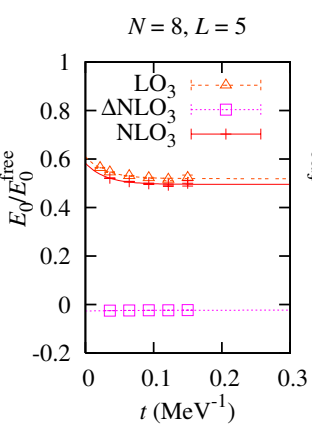

(b)

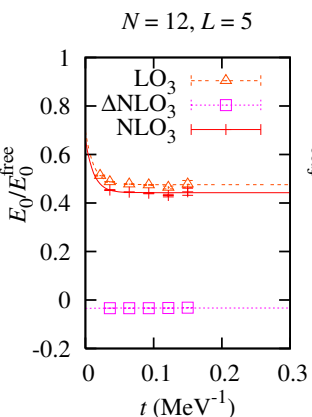

(e)

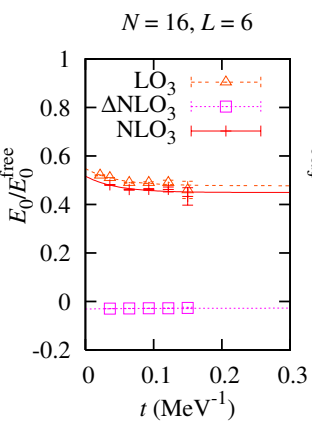

(h)

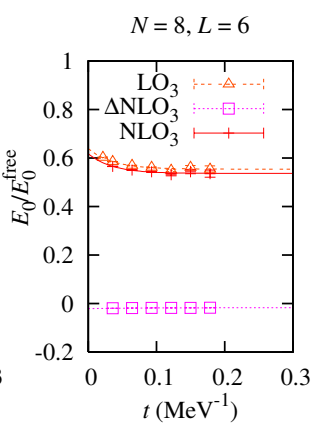

(c)

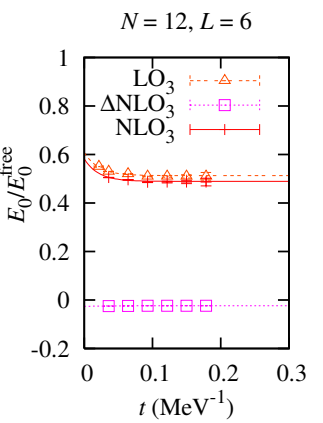

(f)

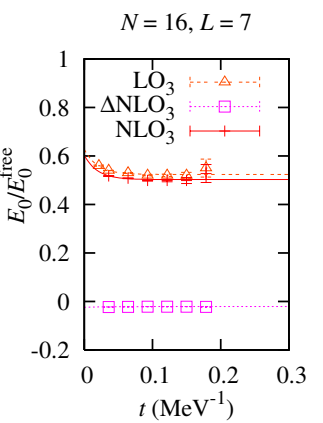

(i)

FIG. 5: Plots of the three energy ratios defined in Eq. (95) versus Euclidean projection time $t$. These are labelled as $\mathrm{LO}_{3}, \Delta \mathrm{NLO}_{3}, \mathrm{NLO}_{3}$ respectively.

\section{DISCUSSION}

\section{A. Comparisons with other results}

In Fig. 6] we compare the ground state energy ratio $E_{0} / E_{0}^{\text {free }}$ for $\mathrm{LO}_{2}, \mathrm{NLO}_{2}, \mathrm{LO}_{3}$, and $\mathrm{NLO}_{3}$ as a function of $k_{F}$. We note two points here. First the difference between $\mathrm{LO}_{3}$ and $\mathrm{NLO}_{3}$ values for $E_{0} / E_{0}^{\text {free }}$ is relatively small over the range of $k_{F}$ plotted. This suggests that the convergence of the effective field theory expansion appears reliable, and the difference 
TABLE IV: Fit results for $E_{0, \mathrm{LO}_{3}} / E_{0}^{\text {free }}$ and $E_{0, \mathrm{NLO}_{3}} / E_{0}^{\text {free }}$.

\begin{tabular}{||c|c|c|c|c|c||}
\hline \hline$N$ & $L$ & $k_{F}(\mathrm{MeV})$ & $E_{0, \mathrm{LO}_{3}} / E_{0}^{\text {free }}$ & $E_{0, \mathrm{NLO}_{3}} / E_{0}^{\text {free }}$ & $\chi^{2} /$ d.f. \\
\hline 8 & 4 & 155 & $0.469(2)$ & $0.436(2)$ & 0.6 \\
\hline 8 & 5 & 124 & $0.519(4)$ & $0.496(4)$ & 0.8 \\
\hline 8 & 6 & 103 & $0.554(4)$ & $0.537(4)$ & 0.8 \\
\hline 8 & 7 & 88 & $0.584(8)$ & $0.571(8)$ & 0.5 \\
\hline 12 & 5 & 142 & $0.476(2)$ & $0.443(2)$ & 1.8 \\
\hline 12 & 6 & 118 & $0.513(2)$ & $0.490(2)$ & 2.0 \\
\hline 12 & 7 & 101 & $0.535(3)$ & $0.518(3)$ & 1.0 \\
\hline 16 & 6 & 130 & $0.477(10)$ & $0.450(10)$ & 1.4 \\
\hline 16 & 7 & 111 & $0.524(3)$ & $0.503(3)$ & 2.0 \\
\hline \hline
\end{tabular}

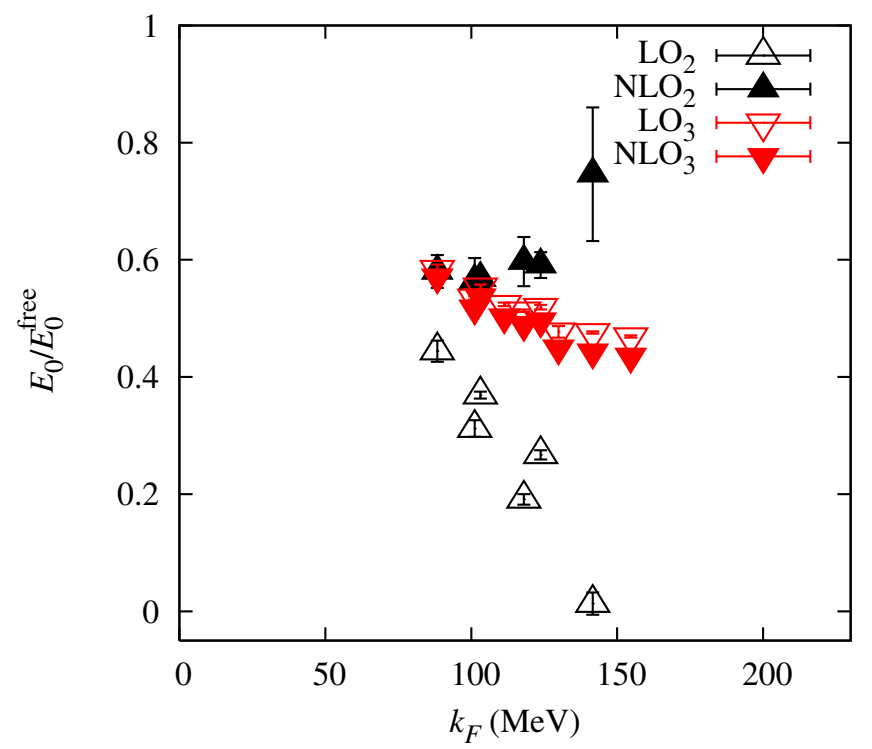

FIG. 6: Comparison of the ground state energy ratio $E_{0} / E_{0}^{\text {free }}$ for $\mathrm{LO}_{2}, \mathrm{NLO}_{2}, \mathrm{LO}_{3}$, and $\mathrm{NLO}_{3}$ as a function of $k_{F}$.

between $\mathrm{LO}_{3}$ and $\mathrm{NLO}_{3}$ values provides an upper estimate on the size of contributions at higher orders. Second the results for $\mathrm{NLO}_{2}$ and $\mathrm{NLO}_{3}$ agree for $k_{F}$ less than $100 \mathrm{MeV}$. This is the region where we expect the perturbative treatment of $\mathrm{NLO}_{2}$ corrections to be accurate. The agreement with $\mathrm{NLO}_{3}$ provides some confidence in the effective field theory 


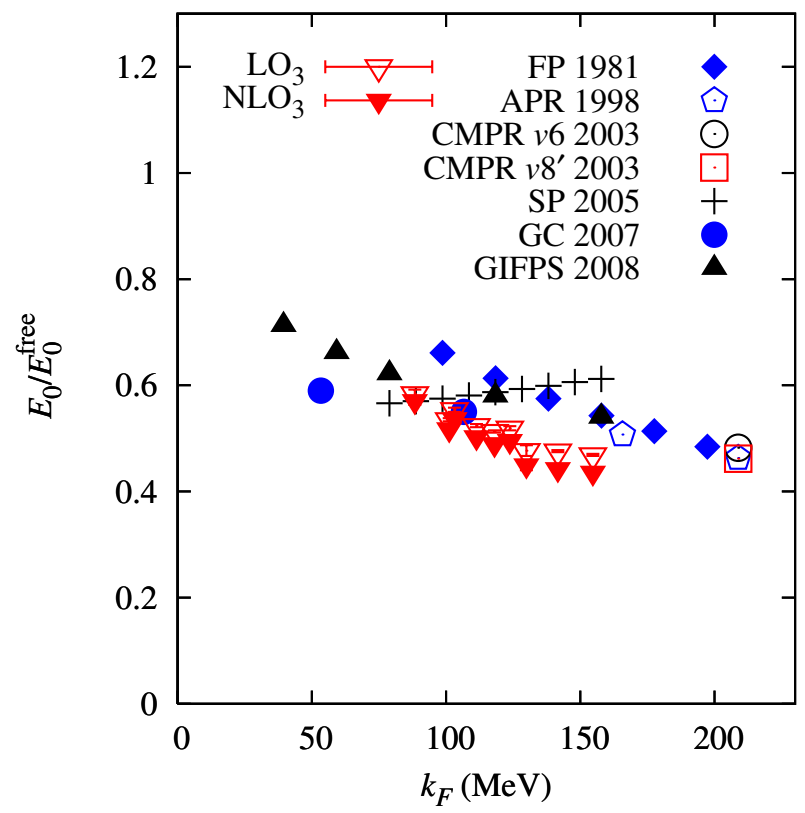

FIG. 7: Ground state energy ratio $E_{0} / E_{0}^{\text {free }}$ for $\mathrm{LO}_{3}$ and $\mathrm{NLO}_{3}$ versus Fermi momentum $k_{F}$. For comparison we show results for FP 1981 [38], APR 1998 [39], CMPR $v 6$ and $v 8^{\prime} 2003$ [40], SP 2005 [41], GC 2007 [42], and GIFPS 2008 [43].

approach to dilute neutron matter. It is also an explicit test of model independence at fixed lattice spacing as suggested in Ref. [4].

In Fig. 7 we compare ground state energies for $\mathrm{LO}_{3}$ and $\mathrm{NLO}_{3}$ with other results from the literature: FP 1981 [38], APR 1998 [39], CMPR $v 6$ and $v 8^{\prime}$ [40], SP 2005 [41], GC 2007 [42], and GIFPS 2008 [43]. Compared with other calculations our ground state energies are slightly lower for $k_{F}$ near $130 \mathrm{MeV}$, but overall there is relatively good agreement.

\section{B. Expansion near the unitarity limit}

The unitarity limit is an idealized limit of attractive two-component fermions where the $S$-wave scattering length is infinite and the range of the interaction is negligible. The $S$ wave scattering length for neutron-neutron scattering is $-18.5 \mathrm{fm}$, while the range of the interaction is comparable to the Compton wavelength of the pion, $m_{\pi}^{-1}$. The unitarity limit is approximately realized in neutron matter when the average particle separation is between these two length scales. This occurs at a Fermi momentum of about $80 \mathrm{MeV}$. In the 
unitarity limit the ground state has no dimensionful parameters other than particle density. Therefore the ground state energy in the unitarity limit should obey a simple and universal relation $E_{0}=\xi E_{0}^{\text {free }}$ for some dimensionless constant $\xi$.

The unitarity limit has been reproduced in trapped cold atom experiments using ${ }^{6} \mathrm{Li}$ and ${ }^{40} \mathrm{~K}$. The scattering length is tuned to infinity using a Feshbach resonance and the system is sufficiently dilute that the range of the interaction is negligible. Recent experimental measurements for $\xi$ give $0.32_{-13}^{+10}$ [44], 0.51(4) [45], $0.46_{-05}^{+12}$ [46], and $0.39(2)$ [47]. There have been numerous analytic calculations for $\xi$ varying over the range from 0.2 to 0.6 [48, 49, 50, 51, 52, 53, 54, 55, 56, 57, 58]. Several numerical calculations both on the lattice and in the continuum find results varying from about 0.25 to $0.45[59,60,61,62,63,64,65,66,67$, 68, 69, 70, 71]. The most recent of these numerical calculations agree on a smaller window between 0.30 and 0.40 .

For finite $S$-wave scattering length $a_{0}$ the deviation away from unitarity can be parameterized as

$$
\frac{E_{0}}{E_{0}^{\text {free }}} \approx \xi-\frac{\xi_{1}}{k_{F} a_{0}}
$$

As shorthand notation we define

$$
f\left(k_{F} a_{0}\right)=\xi-\frac{\xi_{1}}{k_{F} a_{0}}
$$

In the recent literature there is general agreement on the value of $\xi_{1}$, ranging from about 0.8 to $1.0[32,60,66,67,68,62,73]$. In the following analysis we use the values $\xi=0.31$ and $\xi_{1}=0.81$ calculated in Ref. [66].

In addition to the corrections at finite scattering length we expect corrections proportional to $k_{F} r_{0}$ due to the $S$-wave effective range $r_{0}$. For neutron-neutron scattering $r_{0}$ is $2.7 \mathrm{fm}$. We also expect higher-order corrections away from the unitarity limit arising from higher powers of $1 /\left(k_{F} a_{0}\right)$ and $k_{F} r_{0}$, as well as other terms associated with the $S$-wave shape parameter and triplet $P$-wave scattering volumes. In general we can write

$$
E_{0} / E_{0}^{\text {free }} \approx f\left(k_{F} a_{0}\right)+c_{1} k_{F} r_{0}+c_{2} k_{F}^{2} m_{\pi}^{-2}+c_{3} k_{F}^{3} m_{\pi}^{-3}+\cdots
$$

Due to the relatively narrow spread of $k_{F}$ values considered in our lattice simulations, it is difficult to constrain $c_{2}$ and $c_{3}$ and higher coefficient powers. However we can constrain the parameter $c_{1}$. 


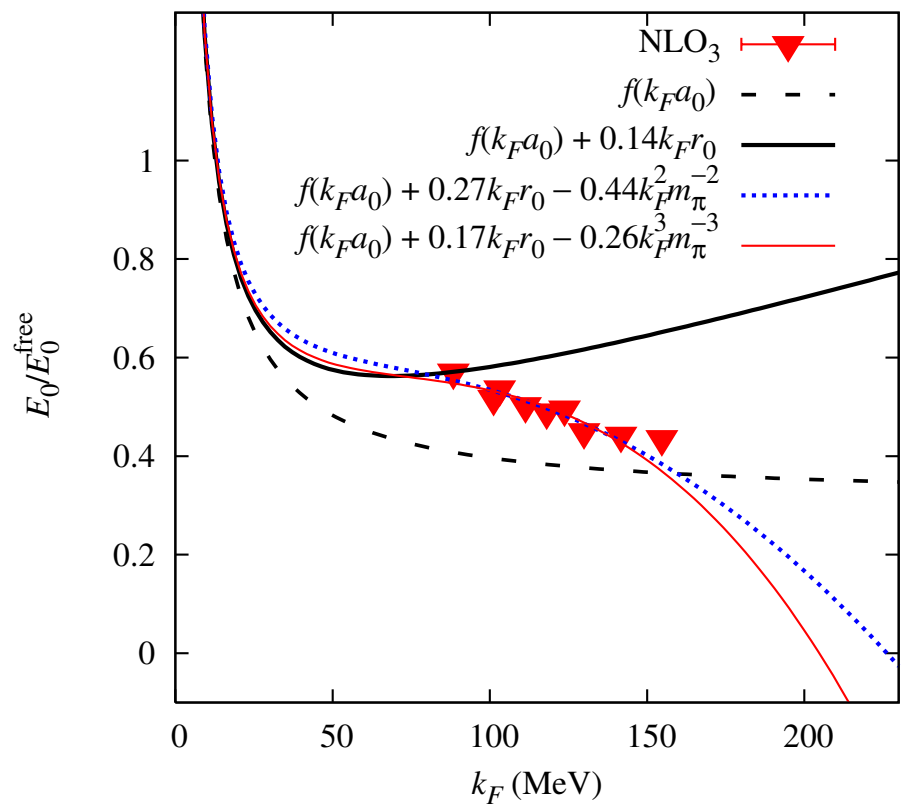

FIG. 8: Comparison of $E_{0, \mathrm{NLO}_{3}} / E_{0}^{\text {free }}$ with various fits involving subsets of the unknown parameters $c_{1}, c_{2}, c_{3}$ as defined in Eq. (102).

If we set $c_{2}=c_{3}=0$ and determine $c_{1}$ from the data point with the smallest value for $k_{F}$ we get

$$
E_{0, \mathrm{NLO}_{3}} / E_{0}^{\mathrm{free}} \approx f\left(k_{F} a_{0}\right)+0.14 k_{F} r_{0}
$$

If instead we set $c_{3}=0$ and determine $c_{1}$ and $c_{2}$ simultaneously we find

$$
E_{0, \mathrm{NLO}_{3}} / E_{0}^{\text {free }} \approx f\left(k_{F} a_{0}\right)+0.27 k_{F} r_{0}-0.44 k_{F}^{2} m_{\pi}^{-2}
$$

As a third alternative if we set $c_{2}=0$ and fit $c_{1}$ and $c_{3}$ simultaneously, we get

$$
E_{0, \mathrm{NLO}_{3}} / E_{0}^{\text {free }} \approx f\left(k_{F} a_{0}\right)+0.17 k_{F} r_{0}-0.26 k_{F}^{3} m_{\pi}^{-3}
$$

The results of these fits are shown in Fig. 8. Our simple analysis suggests a value for $c_{1}$ in the range between 0.14 and 0.27 . This is consistent with the value 0.15 for the same coefficient found in Ref. [6].

\section{SUMMARY}

We have presented lattice simulations for the ground state energy of dilute neutron matter at next-to-leading order in chiral effective field theory. We have solved some problems that 
arose in recent work using leading-order lattice actions $\mathrm{LO}_{1}$ and $\mathrm{LO}_{2}$. $\mathrm{LO}_{1}$ involved pointlike "contact" interactions while $\mathrm{LO}_{2}$ used Gaussian-smeared "contact" interactions. In this work we introduced a new action $\mathrm{LO}_{3}$ which equals $\mathrm{LO}_{2}$ in each $S$-wave channel and equals $\mathrm{LO}_{1}$ in each $P$-wave channel. The action was constructed using projection operators for the spin-singlet/isospin-triplet and spin-triplet/isospin-singlet channels. Using the spherical wall method we computed phase shifts and mixing angles for the new lattice action up to next-to-leading order and fitted all unknown operator coefficients.

In the auxiliary-field formalism we used Euclidean-time projection Monte Carlo to compute the ground state energy of $N=8,12,16$ neutrons in a periodic cube, covering a density range from $2 \%$ to $10 \%$ of normal nuclear density. For $k_{F}$ less than $100 \mathrm{MeV}$ we found ground state energies at next-to-leading order that agreed with earlier lattice results using the action $\mathrm{NLO}_{2}$. For $k_{F}$ greater than $100 \mathrm{MeV}$ we found that the new action leads to much smaller corrections at next-to-leading order. The difference between leading-order and next-to-leading-order values provides an upper estimate on the size of contributions at higher orders. Though we find somewhat lower values for the ground state energy near $k_{F}=130 \mathrm{MeV}$, our results are in general agreement with other calculations reported in the literature.

The ground state energy ratio $E_{0} / E_{0}^{\text {free }}$ was also analyzed as an expansion about the unitarity limit. We considered corrections due to finite scattering length, nonzero effective range, and higher-order effects. If we use the parameterization

$$
E_{0} / E_{0}^{\text {free }} \approx f\left(k_{F} a_{0}\right)+c_{1} k_{F} r_{0}+c_{2} k_{F}^{2} m_{\pi}^{-2}+c_{3} k_{F}^{3} m_{\pi}^{-3}+\cdots
$$

we find $c_{1}$ in the range from 0.14 to 0.27 . In principle the coefficient $c_{1}$ is a universal constant that can be measured in any two-component fermionic system near the unitarity limit. Explicit tests of this universality may be a subject for future investigation. With regard to further investigations of neutron matter, future work on lattice simulations should be considered at next-to-next-to-leading order in chiral effective field theory. Simulations should also be done at smaller and larger lattice spacings to check independence on the lattice spacing and to probe both higher and lower densities. 


\section{Acknowledgements}

We are grateful for discussions with Bugra Borasoy. Partial financial support from the Deutsche Forschungsgemeinschaft (SFB/TR 16), Helmholtz Association (contract number VH-NG-222 and VH-VI-231), and U.S. Department of Energy (DE-FG02-03ER41260) are acknowledged. This research is part of the EU Integrated Infrastructure Initiative in Hadron Physics under contract number RII3-CT-2004-506078. The computational resources for this project were provided by the Jülich Supercomputing Centre at the Forschungszentrum Jülich.

[1] B. Borasoy, E. Epelbaum, H. Krebs, D. Lee, and U.-G. Meißner, Eur. Phys. J. A31, 105 (2007), nucl-th/0611087.

[2] S. Weinberg, Phys. Lett. B251, 288 (1990).

[3] S. Weinberg, Nucl. Phys. B363, 3 (1991).

[4] B. Borasoy, E. Epelbaum, H. Krebs, D. Lee, and U.-G. Meißner, Eur. Phys. J. A35, 343 (2008), arXiv:0712.2990 [nucl-th].

[5] B. Borasoy, E. Epelbaum, H. Krebs, D. Lee, and U.-G. Meißner, Eur. Phys. J. A34, 185 (2007), arXiv:0708.1780 [nucl-th].

[6] B. Borasoy, E. Epelbaum, H. Krebs, D. Lee, and U.-G. Meißner, Eur. Phys. J. A35, 357 (2008), arXiv:0712.2993 [nucl-th].

[7] C. Ordonez and U. van Kolck, Phys. Lett. B291, 459 (1992).

[8] C. Ordonez, L. Ray, and U. van Kolck, Phys. Rev. Lett. 72, 1982 (1994).

[9] C. Ordonez, L. Ray, and U. van Kolck, Phys. Rev. C53, 2086 (1996), hep-ph/9511380.

[10] E. Epelbaum, W. Glockle, and U.-G. Meißner, Nucl. Phys. A637, 107 (1998), nucl-th/9801064.

[11] E. Epelbaum, W. Gloeckle, and U.-G. Meißner, Nucl. Phys. A671, 295 (2000), nuclth/9910064.

[12] J. L. Friar and S. A. Coon, Phys. Rev. C49, 1272 (1994).

[13] N. Kaiser, R. Brockmann, and W. Weise, Nucl. Phys. A625, 758 (1997), nucl-th/9706045.

[14] U. van Kolck, Prog. Part. Nucl. Phys. 43, 337 (1999), nucl-th/9902015.

[15] P. F. Bedaque and U. van Kolck, Ann. Rev. Nucl. Part. Sci. 52, 339 (2002), nucl-th/0203055. 
[16] E. Epelbaum, Prog. Part. Nucl. Phys. 57, 654 (2006), nucl-th/0509032.

[17] E. Epelbaum, H.-W. Hammer, and U.-G. Meißner (2008), arXiv:0811.1338 [nucl-th].

[18] D. B. Kaplan, M. J. Savage, and M. B. Wise, Nucl. Phys. B478, 629 (1996), nucl-th/9605002.

[19] D. B. Kaplan, M. J. Savage, and M. B. Wise, Phys. Lett. B424, 390 (1998), nucl-th/9801034.

[20] D. B. Kaplan, M. J. Savage, and M. B. Wise, Nucl. Phys. B534, 329 (1998), nucl-th/9802075.

[21] S. Fleming, T. Mehen, and I. W. Stewart, Nucl. Phys. A677, 313 (2000), nucl-th/9911001.

[22] S. R. Beane, P. F. Bedaque, M. J. Savage, and U. van Kolck, Nucl. Phys. A700, 377 (2002), nucl-th/0104030.

[23] A. Nogga, R. G. E. Timmermans, and U. van Kolck, Phys. Rev. C72, 054006 (2005), nuclth/0506005.

[24] M. C. Birse, Phys. Rev. C74, 014003 (2006), nucl-th/0507077.

[25] M. C. Birse, Phys. Rev. C76, 034002 (2007), arXiv:0706.0984 [nucl-th].

[26] E. Epelbaum and U.-G. Meißner (2006), nucl-th/0609037.

[27] E. Wigner, Phys. Rev. 51, 106 (1937).

[28] K. Symanzik, Nucl. Phys. B226, 187 (1983).

[29] K. Symanzik, Nucl. Phys. B226, 205 (1983).

[30] V. G. J. Stoks, R. A. M. Kompl, M. C. M. Rentmeester, and J. J. de Swart, Phys. Rev. C48, $792(1993)$.

[31] H. P. Stapp, T. J. Ypsilantis, and N. Metropolis, Phys. Rev. 105, 302 (1957).

[32] D. Lee, Phys. Rev. B75, 134502 (2007), cond-mat/0606706.

[33] D. Lee (2008), arXiv:0804.3501 [nucl-th].

[34] D. Lee, Phys. Rev. C71, 044001 (2005), nucl-th/0407101.

[35] J.-W. Chen, D. Lee, and T. Schäfer, Phys. Rev. Lett. 93, 242302 (2004), nucl-th/0408043.

[36] D. Lee, Phys. Rev. Lett. 98, 182501 (2007), nucl-th/0701041.

[37] D. Lee, Phys. Rev. B73, 115112 (2006), cond-mat/0511332.

[38] B. Friedman and V. R. Pandharipande, Nucl. Phys. A361, 502 (1981).

[39] A. Akmal, V. R. Pandharipande, and D. G. Ravenhall, Phys. Rev. C58, 1804 (1998), nuclth/9804027.

[40] J. Carlson, J. Morales, J., V. R. Pandharipande, and D. G. Ravenhall, Phys. Rev. C68, 025802 (2003), nucl-th/0302041.

[41] A. Schwenk and C. J. Pethick, Phys. Rev. Lett. 95, 160401 (2005), nucl-th/0506042. 
[42] A. Gezerlis and J. Carlson, Phys. Rev. C77, 032801 (2008), 0711.3006.

[43] S. Gandolfi, A. Y. Illarionov, S. Fantoni, F. Pederiva, and K. E. Schmidt, Phys. Rev. Lett. 101, 132501 (2008), 0805.2513.

[44] M. Bartenstein, A. Altmeyer, S. Riedl, S. Jochim, C. Chin, J. Hecker Denschlag, and R. Grimm, Phys. Rev. Lett. 92, 120401 (2004).

[45] J. Kinast, A. Turlapov, J. E. Thomas, Q. Chen, J. Stajic, and K. Levin, Science 307, 1296 (2005), cond-mat/0502087.

[46] J. T. Stewart, J. P. Gaebler, C. A. Regal, and D. S. Jin, Phys. Rev. Lett. 97, 220406 (2006), cond-mat/0607776.

[47] L. Luo and J. E. Thomas (2008), arXiv:0811.1159 [cond-mat.other].

[48] J. R. Engelbrecht, M. Randeria, and C. S. de Melo, Phys. Rev. B55, 15153 (1997).

[49] G. A. Baker, Phys. Rev. C60, 054311 (1999).

[50] H. Heiselberg, Phys. Rev. A63, 043606 (2001), cond-mat/0002056.

[51] A. Perali, P. Pieri, and G. C. Strinati, Phys. Rev. Lett. 93, 100404 (2004).

[52] T. Schäfer, C.-W. Kao, and S. R. Cotanch, Nucl. Phys. A762, 82 (2005), nucl-th/0504088.

[53] T. Papenbrock, Phys. Rev. A72, 041603(R) (2005), cond-mat/0507183.

[54] Y. Nishida and D. T. Son, Phys. Rev. Lett. 97, 050403 (2006), cond-mat/0604500.

[55] Y. Nishida and D. T. Son, Phys. Rev. A75, 063617 (2007), cond-mat/0607835.

[56] P. Arnold, J. E. Drut, and D. T. Son, Phys. Rev. A75, 043605 (2007), cond-mat/0608477.

[57] P. Nikolic and S. Sachdev, Phys. Rev. A75, 033608 (2007), cond-mat/0609106.

[58] M. Y. Veillette, D. E. Sheehy, and L. Radzihovsky, Phys. Rev. A75, 043614 (2007), condmat/0610798.

[59] J. Carlson, S. Y. Chang, V. R. Pandharipande, and K. Schmidt, Phys. Rev. Lett. 91, 50401 (2003), physics/0303094.

[60] G. E. Astrakharchik, J. Boronat, J. Casulleras, and S. Giorgini, Phys. Rev. Lett. 93, 200404 (2004), cond-mat/0406113.

[61] A. Bulgac, J. E. Drut, and P. Magierski, Phys. Rev. Lett. 96, 090404 (2006), condmat/0505374.

[62] E. Burovski, N. Prokofev, B. Svistunov, and M. Troyer, Phys. Rev. Lett. 96, 160402 (2006), cond-mat/0602224.

[63] E. Burovski, N. Prokofev, B. Svistunov, and M. Troyer, New J. Phys. 8, 153 (2006), cond- 
mat/0605350.

[64] D. Lee and T. Schäfer, Phys. Rev. C73, 015201 (2006), nucl-th/0509017.

[65] D. Lee and T. Schäfer, Phys. Rev. C73, 015202 (2006), nucl-th/0509018.

[66] D. Lee, Eur. Phys. J. A35, 171 (2008), arXiv:0704.3439 [cond-mat.supr-con].

[67] T. Abe and R. Seki (2007), arXiv:0708.2523 [nucl-th].

[68] T. Abe and R. Seki (2007), arXiv:0708.2524 [nucl-th].

[69] O. Juillet, New Journal of Physics 9, 163 (2007), cond-mat/0609063.

[70] A. Bulgac, J. E. Drut, and P. Magierski, Phys. Rev. A 78, 023625 (2008), arXiv:0803.3238 [cond-mat.stat-mech].

[71] D. Lee, Phys. Rev. C78, 024001 (2008), arXiv:0803.1280 [nucl-th].

[72] S. Y. Chang, V. R. Pandharipande, J. Carlson, and K. E. Schmidt, Phys. Rev. A70, 043602 (2004).

[73] J.-W. Chen and E. Nakano, Phys. Rev. A75, 043620 (2007), cond-mat/0610011. 